\title{
Improving drought tolerance in rice: Ensuring food security through multi-dimensional approaches
}

\author{
M. Iqbal R. Khan ${ }^{1}$ ( ) | Sudhakar R. Palakolanu ${ }^{2}$ | Priyanka Chopra ${ }^{1}$ | \\ Ashish B. Rajurkar $^{3}$ | Ravi Gupta ${ }^{1}$ | Noushina Iqbal ${ }^{1}$ | Chirag Maheshwari ${ }^{4}$
}

\author{
${ }^{1}$ Department of Botany, Jamia Hamdard, \\ New Delhi, India \\ ${ }^{2}$ Cell, Molecular Biology and Genetic \\ Engineering Group, International Crops \\ Research Institute for the Semi-Arid Tropics \\ (ICRISAT), Hyderabad, India \\ ${ }^{3}$ Institute for Genomic Biology, University of \\ Illinois, Urbana-Champaign, Illinois, USA \\ ${ }^{4}$ Agricultural Energy and Power Division, \\ ICAR-Central Institute of Agricultural \\ Engineering, Bhopal, India

\section{Correspondence} \\ M. Iqbal R. Khan, Department of Botany, Jamia \\ Hamdard, New Delhi, India. \\ Email: iqbal.khan@jamiahamdard.ac.in \\ Funding information \\ UGC-start-up grant, Grant/Award Number: \\ F. $30-482 / 2019$ \\ Edited by: M. Ahanger
}

\begin{abstract}
Drought has been highly prevalent around the world especially in Sub-Saharan Africa and South-East Asian countries. Consistent climatic instabilities and unpredictable rainfall patterns are further worsening the situation. Rice is a $\mathrm{C}_{3}$ staple cereal and an important food crop for the majority of the world's population and drought stress is one of the major growth retarding threats for rice that slashes down grain quality and yield. Drought deteriorates rice productivity and induces various acclimation responses that aids in stress mitigation. However, the complexity of traits associated with drought tolerance has made the understanding of drought stress-induced responses in rice a challenging process. An integrative understanding based on physiological adaptations, omics, transgenic and molecular breeding approaches successively backed up to developing drought stress-tolerant rice. The review represents a step forward to develop drought-resilient rice plants by exploiting the knowledge that collaborates with omics-based developments with integrative efforts to ensure the compilation of all the possible strategies undertaken to develop drought stresstolerant rice.
\end{abstract}

\section{1 | INTRODUCTION}

Climatic fluctuations in recent decades have been escalating the frequency and extremity of calamities in many parts of the world. More than $83 \%$ of the detrimental effects caused by drought occur in the agricultural sector that leads to crop loss and limited productivity, affecting food supplies and livelihood of people and has emerged as

Abbreviations: ABREs, abscisic acid response elements; ACC, 1-aminocyclopropane1-carboxylic acid; APX, ascorbate peroxidase; AQPs, aquaporins; ARFs, auxin response factors; BRs, brassinosteroids; BSA, bulk segregant analysis; CKs, cytokinins; CRT, C-repeat element; DHAR, dehydroascorbate reductase; DRE, dehydration responsive element; DREBs, dehydration responsive element bindings; DEGs, differentially expressed genes; ET, ethylene; ERFs, ethylene response factors; GPOX, glutathione peroxidase; GWAS, genome wide association studies; IAA, indole acetic acid; HSPs, heat shock proteins; LEA, late embryogenesis abundant; MAB, marker-assisted breeding; MABC, marker-assisted backcross; MDA, malondialdehyde; MDHAR, monodehydroascorbate reductase; PBZ, paclobutrazol; RLKs, receptor-like kinases; SA, salicylic acid; SSNs, site-specific nucleases SNP, single-nucleotide polymorphism; TALENs, transcriptional activator-like effector nucleases; TFs, transcription factors; ZFNs, zinc finger nucleases. the most serious famines inducing factor across the globe (FAO, 2018). Rice is one of the major cereals and its consumption comprises over $27 \%$ of total cereal utilisation with an output of 738.2 million tons globally (FAO, 2016). Rice is a paddy field crop with more water requirement for growth and thus drought stress is considered as the major inimical that hinders rice growth, productivity and yield (Mumtaz et al., 2020).

Drought stress detrimentally affects rice production by deteriorating everything from seed germination to the reproductive stages (Pandey and Shukla, 2015; Kumar et al., 2020; Sohag et al., 2020). Drought stress impairs cell growth (Swain et al., 2014), biomass production (Farooq et al., 2010), photosynthesis and increases reactive oxygen species (ROS) accumulation (Sohag et al., 2020) and decreased rice yield (Iseki et al., 2014). Drought also induces a reduction in grain size and weight (Venuprasad et al., 2007) and causes sterility of spikelets (Raman et al., 2012) and consequently leads to a reduction in crop sustainability. Additionally, drought stress severely affects the uptake of nutrients, such as phosphorous (Mumtaz et al., 2020), and their 
acquisition, distribution and storage in plants (Bista et al., 2018). How ever, rice plants acclimate to drought stress through multi-dimensional manipulations at the physiological, molecular and genetical level (Figure 1 and 2).

Consistent efforts are being made to produce drought-tolerant rice varieties with reduced dependency on water availability, improved nutritional status and enhanced grain yield under drought conditions (Gaudin et al., 2013; Selvaraj et al., 2017). The most promising drought-tolerant cultivars may be screened by analysing morpho-physiological and molecular characteristics (Nahar et al., 2018). Additionally, biotechnological interventions successively backed up with omics-based approaches (genomics, proteomics and metabolomics) provide a better insight into understanding the genes associated with drought stress tolerance. Further, at the molecular level, several stress inducible genes mediate drought stress responses in rice through functional proteins or by regulatory elements such as transcription factors (TFs) that control the genes and signal transductions associated with desirable traits under drought stress (Jung et al., 2017; Ganguly et al., 2020; Pan et al., 2020). In facilitating the rice improvement, a detailed understanding of intricate drought stress responses that could aid in the development of rice cultivars more suitable for drought conditions is highly desirable. The advancements in data analysis using omics and gene editing-based approaches have emerged as a valuable addition to pre-existing information of drought tolerance associated traits. The present review is a concerted effort to consolidate major physiological and molecular alterations in rice under drought stress. Additionally, this review discusses the advancements in multi-dimensional approaches for enhancing drought tolerance in rice with a focus on omics tools, genome editing, transgenic development and molecular breeding. The present review also provides a better understanding and possible strategies for developing droughttolerant rice cultivars for sustainable rice production to feed the world population.

\section{2 | PHYSIOLOGICAL MECHANISMS ASSOCIATED WITH RICE ACCLIMATION DURING DROUGHT ASCENDANCY}

\subsection{Root biology}

Roots are the primary plant tissue exposed to drought stress (Niones et al., 2012) and majorly contribute to drought acclimation responses by modulating root architecture and root hydraulic conductivity (Maurel et al., 2010). However, due to the shallow root system in rice, compared to other cereals, it is extremely sensitive to drought conditions (Pandit et al., 2020). Deep-rooting rice cultivars are more resistant to drought than shallow-rooting ones (Uga et al., 2011). Root traits such as root length, rooting depth, root thickness, root length density, root: shoot ratio, number of nodal roots and branching of the

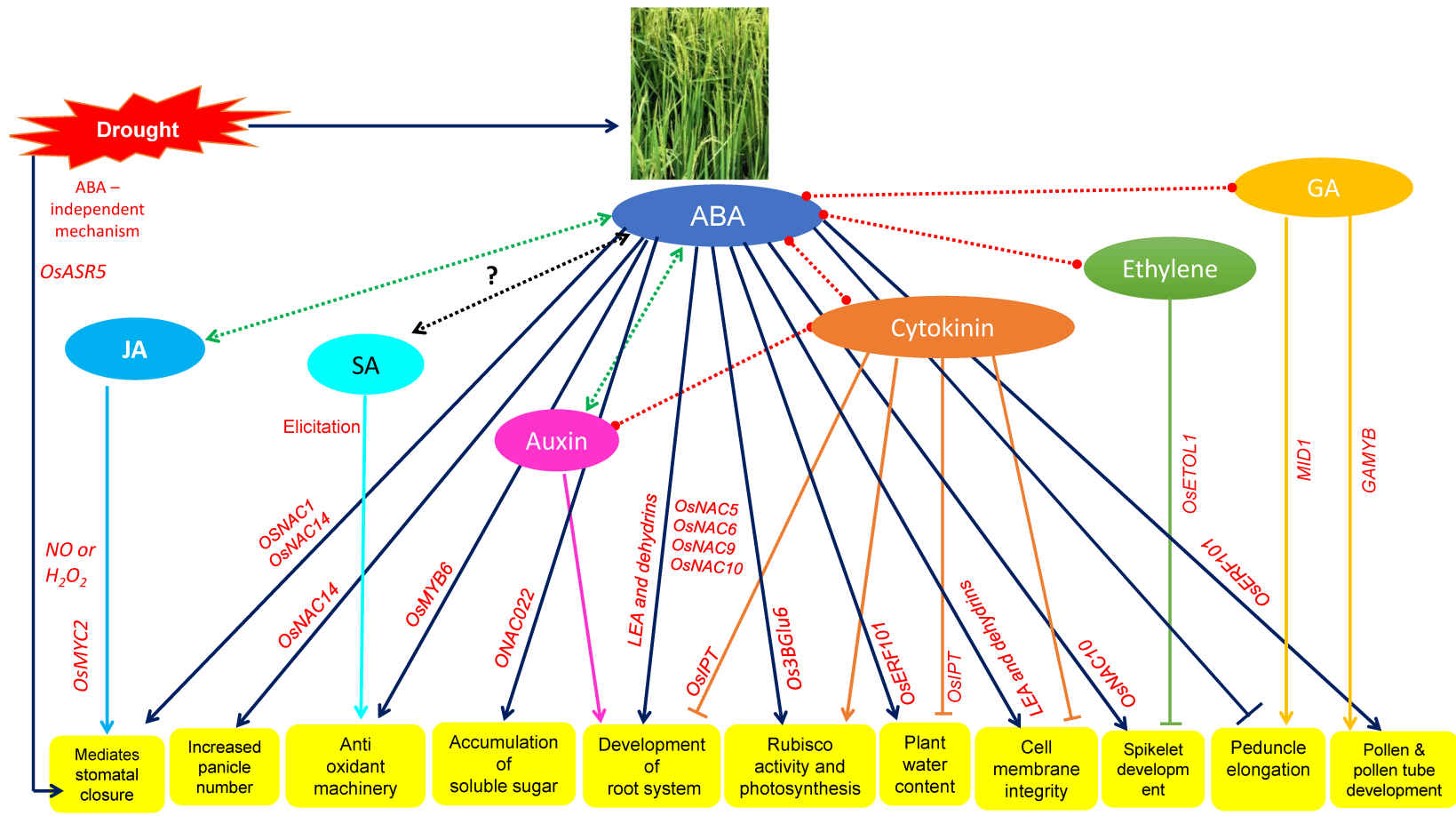

FIGURE 1 Phytohormonal crosstalk in attenuation of rice growth and development during drought stress. During drought conditions in rice, phytohormones act as key mediators to mitigate drought-induced damages and improve plant growth and development. Among all the major plant hormones, ABA act as central mediator for drought stress in rice. Additionally, hormonal-mediated transcription factors also regulate the drought stress mitigation processes in rice. Dotted line with double-sided arrow highlights the positive interaction between the hormones, while dotted lines with rounded heads highlights the negative interaction. T-bars suggests the inhibitory processes, while arrowheads suggest enhanced processes. ABA, abscisic acid 
root systems, are modulated in response to drought conditions (Comas et al., 2013). To prevent drought stress by avoidance mechanism by either growing deeper roots exploiting deep soil moisture or by maintaining dense root growth or by utilising the other traits mentioned needs to be explored (Fukai and Cooper, 1995; Verma et al., 2019). The possession of a deep penetrating, densely branched, thick root system is generally considered favourable for rice plants to enhance its water uptake potential and maintain its water status during drought conditions (Nguyen et al., 1997; Gowda et al., 2011). The rate of water uptake and the cumulative water uptake during the drought stress conditions positively correlates with length, density and thickness of the root system from the OryzaSNP panel of 20 highly diverse rice accessions from Oryza sativa type aus, indica and japonica groups adapted to diverse ecologies and genotyped using single-nucleotide polymorphism (SNP) markers (Gowda et al., 2012). Thus, the water uptake ability of roots of genetically diverse genotypes is an important criterion for understanding drought stress responses in rice.

During drought stress, the plasticity of the root architecture such as total root length, root length density (Tran et al., 2015), lateral root length and branching system (Kano-Nakata et al., 2013) ameliorates stress-induced damages in rice plants. The anatomical features of the rice roots also contribute to the drought stress responses. For instance, cells in the outer parts of the root restrict oxygen loss without hindering water uptake (Ranathunge et al., 2011). The water retention under drought stress is primarily owed to increased suberin development in the endodermis, reduced compaction and suberin formation in sclerenchyma cells, increase in root cross section diameter and reduction in xylem vessel number in rice roots (Henry et al., 2012). Furthermore, plasticity in the architecture of the root system during drought stress is a crucial trait for deciphering the differences within the genotypes in the responses to drought conditions (Henry et al., 2011). During drought conditions, aerenchyma formation negatively regulates root hydraulic conductivity and limits metabolic energy expenditure of the roots thus enhancing drought tolerance (Yang et al., 2012a). Functionally, the plasticity of aerenchyma (root porosity) in rice during drought stress enhances dry weight production and grain yield (Niones et al., 2012). In rainfed lowland rice plants, the spatial distribution of roots largely determines the genetic potential of deeply penetrating roots within the soil to ameliorate drought resistance (Henry et al., 2011). High root-shoot ratio via carbohydrate partitioning, enhanced activities of leaf sucrose-phosphate synthase, root invertase and increased levels of soluble sugars in roots, are some of the major adaptive responses of rice plants to enhance water uptake and to maintain plant's water status for optimal growth during drought (Xu et al., 2015). Thick roots persist longer and produce a heavily branched root system, thereby increasing root length density and water uptake capacity to cope up with drought stress (Ingram et al., 1994). A deep and thick root system is thus the most crucial adaptation of rice plants for improving drought tolerance in a rainfed ecosystem (Comas et al., 2013). Thick roots also have greater root penetration ability in hard soil under drought (Babu et al., 2001).
Aquaporins (AQPs) such as plasma membrane intrinsic proteins including PIP2;6, PIP2;4, PIP2;8 and some root structural parameters in rice such as stele diameter and aerenchyma may possibly affect the hydrostatic and osmotic fluxes of rice roots during drought stress conditions and plant recovery following drought stress (Grondin et al., 2015). Thus, anatomical, cellular, molecular and physiological attributes comprising the root architecture may be altered to enhance water uptake efficiency of the rice plant and aid in the growth and survival of rice plants under drought conditions.

\section{2 | Aquaporins}

AQPs are the intrinsic proteins that primarily form permeable channels for the intracellular transport of water and other solutes to maintain hydraulic conductivity and transpiration demand of the plant (Pawłowicz and Masajada, 2018). APQs are localised in the plasmaand vacuolar membranes, facilitate the passive exchange of water, gaseous molecules such as $\mathrm{O}_{2}$ and $\mathrm{CO}_{2}$, across the membranes, and exhibit a varying specificity for diverse substrates, and the transport is highly regulated (Singh et al., 2020). However, because of the membrane localization of these proteins and the difficulty of solubilising the biological membranes and associated proteins, only a few studies have led to the identification and modulation of AQPs in response to drought stress at the molecular level. Using a label-free quantitative proteomics approach, Mirzaei et al. (2012a) identified 138 proteins that were specifically accumulated in response to extreme drought, 87 proteins that were induced specifically in response to mild drought, and 96 proteins which were specifically present in the well-watered controls in the root proteome of rice leaves (Mirzaei et al., 2012a). Identified proteins included nine AQPs that were induced in response to drought stress then gradually decreased upon re-watering. In rice, a total of 33 AQPs belonging to the four major subfamilies, that is, PIP, TIP, NIP and SIP were identified (Sakurai et al., 2005). Although systematic genome-wide analyses have been done for the AQP family genes in rice, functional validation studies are less reported. Until now, few AQP genes have been functionally characterised in rice in response to drought stress. The rice water channel 3 (RWC3/ OsPIP1;3) protein with stress inducible promoter plays a crucial role in drought avoidance in transgenic lowland rice (Lian et al., 2004). Overexpression of either OsPIP1 or OsPIP2 in Arabidopsis thaliana confers salt and drought stress tolerance (Guo et al., 2006). In addition, transgenic Arabidopsis overexpressing OsPIP1 and OsPIP2 showed higher hydraulic conductivity levels, higher survival rates under drought conditions and increased tolerance to arsenite and higher biomass accumulation, respectively (Li et al., 2016). OsPIP2 overexpression and RNAi studies in rice showed that the root hydraulic conductivity (Lpr), stomatal conductance, root parameters and intercellular $\mathrm{CO}_{2}$ concentrations increase in plants overexpressing OsPIP2 while decreases in RNAi knock-down plants (Ding et al., 2019). Overexpression of OsPIP2;4 in two different rice cultivars depend on the plant genetic architecture to drought stress (Nada and Abogadallah, 2020). Thus, AQPs are involved in the movement of 
water molecules across the membrane and contribute to the regulation of water fluxes throughout the rice plant during drought stress conditions.

\section{3 | Mineral nutrients}

Ensuring efficient rice production with adequate nutritional quality is of paramount importance as rice is a major source of consumption for more than half of the total world's population. Therefore, when rice plants are exposed to drought conditions, the degraded nutritional quality is the major concern among all the other detrimental effects. Even under nutrient rich soil conditions, drought stress influences the nutrient mobility and uptake by the plant and their translocation to the leaves (Bista et al., 2018). Drought stress reduces the availability of nutrients in soil matrix and lowers the transportation in the plant tissue (Silva et al., 2011). Plant roots are primarily involved in nutrient and water uptake and are considered as the key organ to be affected during drought conditions (Koevoets et al., 2016; Kaashyap et al., 2018). Therefore, root architecture is the major factor for maintaining nutritional quality in rice, for instance root hairs are the limiting determinant for nutrient uptake without influencing uptake of water at the seedling stage (Suzuki et al., 2003).

The reduction in transpiration rates is correlated with decrease in nutrient uptake during drought conditions (Yambao and O'Toole, 1984). Under drought stress, nutrient transportation is also severely affected due to the decreased activities of several nutrient transporter proteins such as NRT1, AMT1, PHT1 which further leads to lower nutrient accumulation within the root and shoots of rice (Bista et al., 2018). Drought stress changes the nutritional status of the plant which in turn leads to multiple physiological and metabolic alternations in rice plants. Significant reductions in silicon (Si) levels were observed in the root but not in leaves of rice during drought stress with dreadful effects on root architecture including length, volume, growth and activity of the root system (Chen et al., 2011). Drought stress had adverse effects on potassium $(\mathrm{K})$ nutrient levels within the plant and deteriorated plant physiological functions such as photosynthesis, transpiration and stomatal conductance in rice (Mumtaz et al., 2020).

Drought stress also alters carbon (C) distribution and assimilation in plants (Feller, 2016). Plant metabolism is highly restricted during drought stress conditions that ultimately lowers the C-fixation rate and reduces $\mathrm{CO}_{2}$ assimilation rates in the leaves (Lawlor, 2002). Other drought-induced adversities such as stomatal closure, disruption in membrane integrity and disturbance in activities of enzymes associated with ATP further reduce C-assimilation in the leaves (Dash et al., 2018). Moreover, drought-induced ROS accumulation and oxidative damage to the plants primarily leads to low $\mathrm{CO}_{2}$ fixation that further disturbs the $\mathrm{C}: \mathrm{N}$ ratio and reduces photosynthesis in rice but increases the photorespiration to alleviate oxidative damage (Dusenge et al., 2019). However, drought-induced photorespiration further enhances nitrogen $(\mathrm{N})$ uptake in plants and $\mathrm{N}$ may further enhance photosynthetic machinery during the early developmental stage in rice (Guo et al., 2007).
Both micro and macro-nutrients have an important role in improving drought stress tolerance in plants and supplementation of nutrients may alleviate drought-induced damages (Bardhan et al., 2018; Elbasan et al., 2020) through maintaining redox homeostasis, leaf gas exchange and increased osmolyte accumulation in rice (Ming et al., 2012; Zain and Ismail, 2016). Among various nutrients, $\mathrm{K}$ has an important role in maintaining plant-water relations and stomatal activity (Hasanuzzaman et al., 2018). It has been reported that highaffinity $\mathrm{K}$ transporters such as KT/HAK/KUP induce drought stress resilience by maintaining $\mathrm{K}$ homeostasis in rice (Bañuelos et al., 2002; Yang et al., 2014; Chen et al., 2017). In agricultural soil, K modulates root architecture by increasing the surface area of the root system to facilitate increased absorption of minerals and nutrients (Bardhan et al., 2018). A significant increase in $\mathrm{K}^{+}$levels can enhance various physiological and biochemical parameters such as antioxidant enzyme activities, stomatal conductance, nutrient uptake, water use efficiency and leaf traits associated with mitigation of drought stress damages in plants (Wang et al., 2013). Hyper elevated levels of $\mathrm{K}^{+}$due to the application of $\mathrm{KCl}$ or $\mathrm{K}_{2} \mathrm{SO}_{4}$ lead to increased transpiration, net assimilation rates, proline content and lipid peroxidation along with a significant decrease in antioxidant enzymatic activity of catalase (CAT) (Zain and Ismail, 2016). Calcium (Ca) in the form of $\mathrm{CaCl}_{2}$ enhanced drought stress tolerance in the rice varieties Subhadra and Ghanteswari more efficiently than Sidhant, Jogesh and Khandagir varieties of rice at panicle initiation stage by enhancing the photosynthetic rate, stomatal conductance, leaf moisture retention and membrane stability index, as well as grain yield (Devi and Kar, 2013). Moreover, treatment of rice plants with $\mathrm{N}$ in the form of $\mathrm{N}$-containing compounds such as ammonium $\left(\mathrm{NH}_{4}{ }^{+}\right)$and nitrate $\left(\mathrm{NO}_{3}{ }^{-}\right)$enhanced drought tolerance to a different extent by mediating AQPs and water and mineral uptake (Ding et al., 2015). For example, $\mathrm{NH}_{4}{ }^{+}$treatment in rice enhanced the expression of AQPs and the roots' ability for water and nutrients uptake to a greater extent compared to that of $\mathrm{NO}_{3}{ }^{-}$treatment, thereby mediating drought tolerance (Ding et al., 2015). Guo et al. (2007) reported that, rice seedlings were treated with $\mathrm{NO}_{3}{ }^{-}$and $\mathrm{NH}_{4}{ }^{+}$as a source of $\mathrm{N}_{\text {. }} \mathrm{NH}_{4}{ }^{+}$treatment enhanced the $\mathrm{N}$ content and the photosynthetic rate increasing the drought tolerance whereas no such observation was noted in case of seedlings treated with $\mathrm{NO}_{3}{ }^{-}$. These studies can be utilised to formulate an efficient fertiliser plan aiding in the improvement of rice.

Similarly, despite being a non-essential mineral element in plants, $\mathrm{Si}$ is considered as a plant beneficial element that alleviates drought stress in rice plants (Ming et al., 2012). Selenium (Se) is an essential trace element in plants, which has a role in improving the grain quality and nutritional status of rice under drought stress (Emam et al., 2014). Pre-treating two rice cultivars (Giza 177 and ET 1444) with Se and Si in the form of sodium selenite $(0.03 \mathrm{mM})$ and potassium silicate $(1.5 \mathrm{mM})$, respectively, under water deficiency improved grain quality and plant growth. The application of Se and Si also improved the nutritional quality of rice by increasing $\mathrm{Ca}-$, iron- ( $\mathrm{Fe}$ ) and P-levels in rice plants (Emam et al., 2014). Scandium (Sc), a rare earth element may also enhance drought stress tolerance in rice by alleviating the deterioration of stomatal conductance, C-assimilation rate, 
transpiration rate, and intracellular $\mathrm{CO}_{2}$ concentration and induce the activity of antioxidant enzymes such as (peroxidase [POD], glutathione reductase [GR], glutathione S-transferase [GST], monodehydroascorbate reductase [MDHAR], dehydroascorbate reductase [DHAR]) (Elbasan et al., 2020). However, Sc treatment during combined drought and salt stress enhanced the activity of antioxidant enzymes and ascorbate (Elbasan et al., 2020). Thus, manipulation of nutrient uptake and nutrients supplementation may aid in mitigating drought stress-induced damages in rice plants.

\section{4 | Phytohormones}

Drought stress induces several plant stress responses to ensure their survival in unfavourable environmental conditions. Among several stress-coping strategies, phytohormones-mediated drought tolerance is of utmost importance. Phytohormones are involved in mediating drought stress responses in rice plants (Figure 1).

Drought-induced responses depend on the interactome of different signal transduction pathways (de Ollas and Dodd, 2016). After the drought perception, the activated molecules generate hormonal and chemical signals that further may interact with each other to mediate the drought stress responses (Kazan, 2015). Among all the major phytohormones, abscisic acid (ABA) is considered as the key mediator in controlling water loss and plant water levels during drought stress (de Ollas and Dodd, 2016; Kuromori et al., 2018; Zhang et al., 2020a)
(Table 1). Endogenous production of ABA increases within the plant system in response to several stress cues, such as drought (Vishwakarma et al., 2017). In rice, ABA modifies root growth for enhancing water uptake during drought stress (Zhang et al., 2020a) as well as enhances the accumulation of soluble sugars for maintaining leaf water content and osmotic balance (Pattanagul, 2011).

During drought stress conditions, ABA accumulates in a significant amount and mediate cellular, physiological and molecular responses. Additionally, it coordinates stress-induced signal transduction pathway in rice plants. Among these responses, stomatal movement is an important mechanism, along with the induction of drought stress related genes, accumulation of ROS and metabolites such as soluble sugars (Ye et al., 2012; Dash et al., 2018). Stomatal closure is an ABA-mediated mechanism that efficiently reduces water loss, (Kim et al., 2010), by altering the ion homeostasis of the guard cells (Kim et al., 2010). Molecular and genetic insights have suggested that the expression of drought stress responsive genes involves regulatory systems that may be $A B A$ dependent or $A B A$ independent (Riera et al., 2005; de Ollas and Dodd, 2016). Various ABA responsive genes significantly control drought stress responses for optimal plant growth during water deficiency. For instance, ABSCISIC ACID STRESS RIPENING (ASR) genes that consist of an ABA/WDS domain have been identified from in both monocots and dicots (Gonzalez and lusem, 2014). These genes have a prominent role in ABA-mediated stress responses in rice. The OSASR1 gene induces the drought stress responses by reducing stress-induced phenotypic alterations and enhancing plant

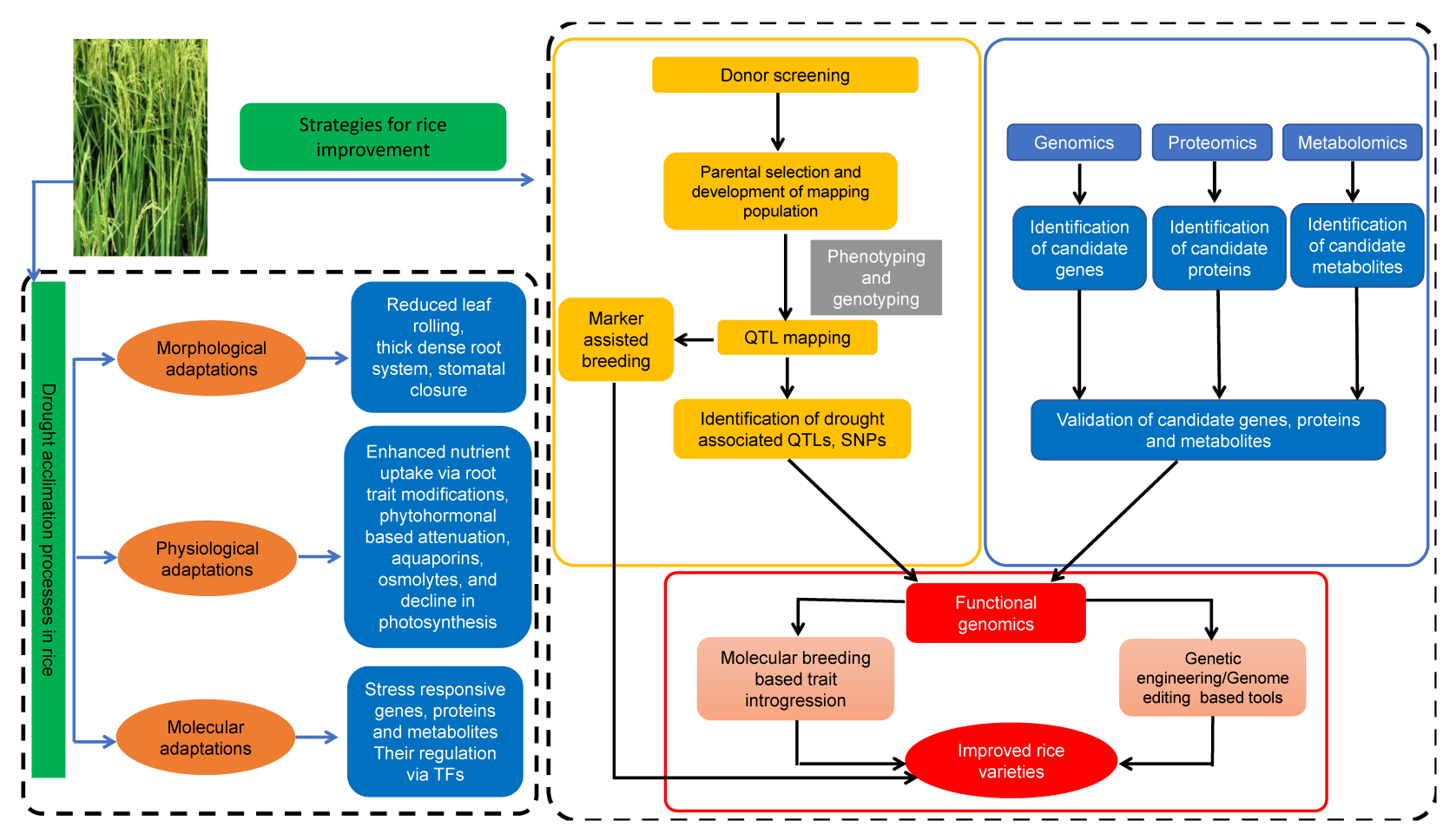

FIGURE 2 Acclimation processes of rice during drought stress and rice improvement strategies. Rice plants acclimate in response to drought stress conditions by inducing various physiological, molecular and genetic manipulations. Rice improvement under drought stress involves molecular breeding and genetic engineering-based approaches. However, both approaches are backed up well by omics-based tools for improving rice during drought stress 
TAB LE 1 Role of abscisic acid (ABA) and its crosstalk with other phytohormones during drought stress

\begin{tabular}{|c|c|c|c|}
\hline Effect on ABA & Effect on other Phytohormones & $\begin{array}{l}\text { Interaction level (response of genes } \\
\text { or activity) }\end{array}$ & References \\
\hline Increased accumulation of ABA & Modulates auxin transport in root tip & $\begin{array}{l}\text { Plasma membrane } \mathrm{H}^{+} \text {-ATP ase activity } \\
\text { increased to increase Root growth and } \\
\text { root hair development }\end{array}$ & Xu et al., 2013 \\
\hline $\begin{array}{l}\text { ABA-deficient mutant or floridone } \\
\text { treatment }\end{array}$ & $\begin{array}{l}\text { Auxin biosynthetic genes decreased. IAA } \\
\text { (OsYUCCA1, OsYUCCA2, OsYUCCA4, } \\
\text { OsYUCCA7, OsOASA1 and OsTDD1 }\end{array}$ & $\begin{array}{l}\text { This decrease in ABA and auxin causes } \\
\text { drought sensitivity }\end{array}$ & Du et al., 2013 \\
\hline $\begin{array}{l}\text { JERF1 induces ABA biosynthesis } \\
\text { enzyme gene (OsABA2 and } \\
\text { Os03g0810800) }\end{array}$ & $\begin{array}{l}\text { JERF1 (ethylene response factor) was } \\
\text { induced by ABA and it acted on stress } \\
\text { related genes }\end{array}$ & $\begin{array}{l}\text { Overexpression of JERF1 induced OsP5CS } \\
\text { and stress related genes to provide } \\
\text { drought tolerance }\end{array}$ & Zhang et al., 2010 \\
\hline $\begin{array}{l}\text { OsDERF1gene is induced by ABA } \\
\text { and drought }\end{array}$ & $\begin{array}{l}\text { OsDERF1 binds ERF genes and decrease } \\
\text { ethylene synthesis }\end{array}$ & $\begin{array}{l}\text { Decreased ethylene because of ERF } \\
\text { repressor OsERF3 and OsAP2-39 } \\
\text { activated by OsDERF1-ERF decreased } \\
\text { drought tolerance }\end{array}$ & Wan et al., 2011 \\
\hline $\begin{array}{l}\text { OsAP2-39 (APETALA } 2 \\
\text { transcription factor)induces } \\
\text { ABA biosynthetic gene } \\
\text { (OsNCED1) }\end{array}$ & $\begin{array}{l}\text { OsAP2-39 induces GA catabolic gene } \\
\text { ELONGATION OF UPPER MOST } \\
\text { INTERNODE I (EUI) }\end{array}$ & $\begin{array}{l}\text { Overexpression of OsAP2-39 did not cause } \\
\text { drought tolerance }\end{array}$ & Yaish et al., 2010 \\
\hline \multirow[t]{2}{*}{$\begin{array}{l}\text { SL deficiency in rice stimulates } A B A \\
\text { accumulation in shoots }\end{array}$} & $\begin{array}{l}\text { OsD27 expression is increased by SL } \\
\text { deficiency }\end{array}$ & $\begin{array}{l}\text { Increased } A B A \text { led to drought tolerance } \\
\text { which is mediated by OsD27 }\end{array}$ & Haider et al., 2018 \\
\hline & Induced by both $\mathrm{JA}$ and $\mathrm{ABA}$ & $\begin{array}{l}\text { jasmonate (MeJA) is produced which in turn } \\
\text { stimulates } A B A \text { formation leading to loss } \\
\text { of grain yield }\end{array}$ & Kim et al., 2009 \\
\hline $\begin{array}{l}\text { OsJAZ1 (JAZ, JASMONATE ZIM- } \\
\text { domain) might repress ABA } \\
\text { signalling }\end{array}$ & It also represses JA signalling & $\begin{array}{l}\text { It shows increased root and shoot length } \\
\text { but decrease drought tolerance }\end{array}$ & Fu et al., 2017 \\
\hline
\end{tabular}

Note: The table describes the role of ABA as a central drought hormone along with its crosstalk with other phytohormones ad their effect on drought responsive traits of the rice plant.

survival rate (Park et al., 2020). OsASR5 may enhance drought tolerance in rice by mediating leaf water content via regulation of stomatal movements (Li et al., 2017). Wang et al. (2020a) reported that the chloroplastic Os3BGlu6 gene is induced by both $A B A$ and drought in rice and regulates the $A B A$ response in order to increase the Rubisco activity and photosynthesis under drought stress.

In addition to ABA-dependent genes, the expression of some drought-induced genes is ABA-independent (Yamaguchi-Shinozaki and Shinozaki, 2005). These 'drought-inducible genes', which are induced independent of ABA, have a conserved 'dehydration responsive element' (DRE) or C-repeat element (CRT) in their promoter region that is stimulated by external stimuli (Yamaguchi-Shinozaki and Shinozaki, 2005). Dehydration responsive element binding proteins (DREBs) are important transcription factors (TFs) that mediate drought stress tolerance and binds to DRE/CRT cis-elements to regulate the expression of stress responsive genes during drought conditions (Sakuma et al., 2006). This activates genes that encode protein kinases, dehydrins, late embryogenesis abundant (LEA) proteins, heat shock proteins (HSPs) and starch degrading enzymes. LEA proteins and dehydrins are crucially involved in mitigating drought stress conditions by binding water, sequestering ions, protection of protein structure and membrane integrity (Hanin et al., 2011; Duan and Cai, 2012).
Deciphering the intricate molecular machinery of ABA-mediated responses, their signalling and perception may further enhance the understanding of plant adaptation to drought stress (Umezawa et al., 2010). The regulatory system for ABA responses majorly consists of receptors PYR/RCAR/PYL, negative regulators such as PP2C, and positive regulators such as SnRK2 that interact with each other during drought stress, to mediate drought tolerance (Ma et al., 2009; Park et al., 2009; Dittrich et al., 2019; Takahashi et al., 2020). The PYLs function excessively in the presence of the co-receptors PP2Cs, for example, MYC2 (a regulator of JA signalling) is mediated by PLYs to show interactive effects on inhibition of seed germination (Aleman et al., 2016). In addition, ABA dependent SnRK2.6 is the major regulator of ion flux through guard cells and is involved in stomatal closure mediated by an anion channel, SLAC1; transporters such as KUP6 and ALMT12 (Chen et al., 2020). However, SnRK2-independent regulation of guard cell functioning and stomatal closure is mediated by calcium protein kinases (Pornsiriwong et al., 2017).

Several drought responsive genes may have additional regulatory motifs in their promoter regions such as ABA-response elements (ABREs) connecting different stress-associated regulatory pathways (Nakashima and Yamaguchi-Shinozaki, 2006; Srivasta et al., 2010). Similar to binding with DRE/CRT elements of ABA, DREB promoters 
also shows co-localised motifs for auxin signalling and binds to the auxin response factors (ARFs). This trio of ABA, auxin and DREB opens up a crosstalk between $A B A$ and auxin-mediated drought tolerance in rice via DREB (Srivasta et al., 2010). There may be a possible crosstalk between $A B A$ and auxin at the biosynthesis level that mediates plant development and enhances drought tolerance in rice (Du et al., 2013). Carotenoid deficient rice mutants (PDS-RNAi transgenic rice and phs1, phs2, phs3-1, phs4) with impaired biosynthesis of both $A B A$ and indole acetic acid (IAA) exhibited larger stomata and more wilting than the wild-type (Du et al., 2013). Additionally, inhibition of $A B A$ by fluoride (ABA biosynthesis inhibitor) caused reduced IAA levels by downregulating the expression of genes related to its biosynthesis and metabolism. These regulations show the genetic control of stress and pinpoint interaction between hormones for regulating drought tolerance. ABA-induced stomatal closure is the earliest drought stress response to mitigate the excess loss of water in plants (Schroeder et al., 2001) and may be influenced by/or interact with other phytohormones to mediate stomatal closure (DaszkowskaGolec and Szarejko, 2013). Many reports strongly suggest the involvement of jasmonic acid (JA) in ABA-mediated stomatal closure along with other molecules such as nitric oxide (NO) during water deficiency, however, the reported involvement of phytohormones are such as cytokinin (CK), auxins and ethylene (ET) in stomatal movement is ambiguous (Huang et al., 2008; Daszkowska-Golec and Szarejko, 2013; Shi et al., 2014). JA is involved in ABA-induced stomatal closure as it enhances the influx of extracellular $\mathrm{Ca}^{2+}$ that stimulates the production of Ca-dependent protein kinases and/or activates $\mathrm{NO}$ or $\mathrm{H}_{2} \mathrm{O}_{2}$ signalling (Harrison, 2012). Nitric oxide also acts as a key signalling molecule that regulates $A B A$-mediated stomatal closure in plants. ABA stimulates the production of $\mathrm{NO}$ that further elicits closure of stomata during drought conditions by promoting efflux of anions from guard cells and inactivation of inward rectifying cationic channels (Laxalt et al., 2016; Sun et al., 2019). CK and ABA signalling undergo an antagonistic crosstalk with each other that fine tunes plant growth and drought stress responses as observed in Arabidopsis (Huang et al., 2018). CK negatively regulates drought stress signalling in plants as is directly evident by the high-water content maintaining capacity, cell membrane integrity and ABA hypersensitivity seen in CK deficient Arabidopsis plants during drought tolerance (Nishiyama et al., 2011). The exogenous ABA-mediated alteration in expression of genes involved in CK biosynthesis and metabolism (such as ISOPENTENYL-TRANSFERASE and CYTOKININ OXIDASES/DEHYDROGENASES) raises the possibility that $C K$ might be involved in a crosstalk with $A B A$ to mediate drought-induced stress responses in plants (Nishiyama et al., 2011). Like auxins, CKs also have an inconclusive participation in drought-induced stomatal closure in plants (Daszkowska-Golec and Szarejko, 2013). Ethylene is also involved in an antagonistic crosstalk with $A B A$ via novel ET response factors (ERF) transcriptional cascade that mediates drought stress responses by regulating ET biosynthesis in rice (Wan et al., 2011). Rice seedlings overexpressing the ERF repressors OsERF3 and OsAP2-39 has shown decreased expression of ET biosynthesis genes suppressing ET production (Wan et al., 2011). Moreover, 1-aminocyclopropane-1- carboxylic acid (ACC) application to drought sensitive phenotypes in overexpressed lines recovered drought tolerance which suggests the involvement of $A B A$ and drought-induced ERFs in reducing ET production and enhancing drought stress tolerance in rice (Wan et al., 2011). ERF repressor OsERF3-mediated ET production and drought tolerance in rice is possibly regulated by amino acid residues flanking the EAR motifs (Zhang et al., 2013). EAR motifs are the transcriptional repressor motifs with a patterned consensus sequence that negatively regulate plant responses to drought stress (Zhang et al., 2013) Also, an ERF protein from tomato, that is, JERF1 may also regulate the drought stress response in transgenic rice via an $A B A$ pathway as indicated by the increased ABA levels in JERF1 overexpressed transgenic lines of rice. The antagonistic crosstalk between $A B A$ and ET may also mediate the effect of drought stress on spikelet fertility in rice during meiosis as during water stress (Yang et al., 2007). Enhanced ABA production and reduced ET levels promotes spikelet growth in rice during meiosis in drought conditions and thus a higher ratio of $A B A$ to $A C C$ or $A B A$ to $E T$ are significant for drought stress tolerance in rice (Yang et al., 2007). Conclusively, ABA although the central drought stress mediating phytohormone in rice, its molecular and physiological crosstalk with other phytohormones suggest that it is a part of an intricate network for drought stress tolerance in rice (Seo et al., 2011; de Ollas and Dodd, 2016).

Phytohormones other than ABA also contribute individually in drought stress tolerance in rice. For example, auxin is pivotal for root development and thus any intervention with its biosynthesis and signalling can adversely hinder the development of root system of rice (Zhao et al., 2015). Exogenous application of IAA (10 $\left.{ }^{-5} \mathrm{M}\right)$ mitigates stress-induced adversities on yield, spikelet fertility, and pollen viability, significantly during drought and heat stresses in rice (Sharma et al., 2018). Conversely, auxin may also negatively regulate the genes involved in drought stress avoidance in rice as instanced by phenotypic and molecular characterisation of the DEEPER ROOTING 1 (DRO1) gene involved in drought stress avoidance by enhancing root growth angle mediating higher root growth descending in the soil (Uga et al., 2013). The overexpression of auxin efflux carrier genes such as OsPIN3t (Zhang et al., 2012), OsGH3.2 (Du et al., 2012), OsGH3.13 (Zhang et al., 2009) improves drought stress tolerance in rice. The levels of $\mathrm{CK}$ also changes during drought stress by the virtue of alteration in expression of IPT (isopentenyl transferase) genes involved in CK biosynthesis as instanced in Arabidopsis and rice (Ghosh et al., 2018). CK enhances drought tolerance through the coordinated regulation of $\mathrm{C}$ and $\mathrm{N}$ assimilation by IPT genes (Reguera et al., 2013). Expression of IPT gene in transgenic rice under the control of PSARK, stress- and maturation-induced promoter enhanced drought tolerance. It also increased grain yield with improved grain quality under drought stress by upregulation of brassinosteroids (BRs) associated genes and repression of JA associated genes resulted in alternation of hormonal homeostasis and source sink relationship in rice lines (Peleg et al., 2011). However, a negative interaction between auxin and $\mathrm{CK}$ signalling may also control drought stress mitigating mechanisms such as rice crown root formation via OsCKX4, a CK metabolism gene in rice (Gao et al., 2014). Confirmation of OsARF25 
and the OsCKX4 through yeast one hybrid assay suggested the role of OsCKX4 in auxin signalling, while overexpression of OsCKX4 reduced auxin levels while knockdown mutants of OsCKX4 enhanced auxin levels in rice suggesting $\mathrm{CK}$ to be an important regulator of auxin biosynthesis (Gao et al., 2014). ET is also involved in regulation of drought stress responses in rice via ERFs. For example, overexpression of JERF1 activates stress responsive genes such as OsP5CS and OsSPDS2 (osmolyte synthesis), increases proline accumulation in transgenic rice and enhances drought tolerance at the seedling stage (Zhang et al., 2010). Overexpression of OsERF109 decreases drought stress tolerance characterised by rolled and wilted leaves, rapid water loss and decreased survival rates of the plants (Yu et al., 2017). Furthermore, the expression of ET associated genes such as OsACS6 and OsACO2 also decreased. The role of ET in drought stress adaptation in rice via ETO genes is also suggested. The osetol1 mutant of ETHYLENE OVERPRODUCER 1-like gene (OsETOL 1) resulted in enhanced spikelet fertility and biomass at the reproductive stage during drought stress in rice (Du et al., 2014). However, OsETOL1 negatively regulates ACC production and ET biosynthesis, and OsETOL1 overexpressed plants exhibit decreased spikelet fertility (similarly to ACC deficient mutants), delayed senescence and reduced carbohydrate transportation from leaves to seeds and drought stress tolerance (Du et al., 2014). The role of gibberellin (GA) in altering drought stress tolerance in rice plants has been suggested by the semi-dwarf rice mutants that were deficient in GA biosynthesis and exhibited enhanced drought tolerance (Plaza-Wüthrich et al., 2016). Deficiency of GA or inhibition in its formation leads to the development of semidwarf wheat and rice cultivars with significantly high yield during the green revolution (Hedden, 2003). Paclobutrazol (PBZ) is a GA inhibitor and its application in Japanese paddy rice increased the yield although plant height was reduced by $90 \%$ (French et al., 1990). Semi-dwarf rice mutants deficient in GA biosynthesis were found to be drought tolerant (Plaza-Wüthrich et al., 2016) suggesting lower GA are required for drought tolerance. Salicylic acid (SA) and $\mathrm{H}_{2} \mathrm{O}_{2}$ enhances the activities of antioxidant enzymes such as CAT, ascorbate peroxidase (APX) and glutathione peroxidase (GPX) and enhances the levels of osmolytes such as proline resulting in osmotic adjustments for better survivability of rice plant under drought conditions (Sohag et al., 2020). In nutshell, phytohormones mediate drought stress tolerance in rice and regulate its growth and development either individually or/and via crosstalk with each other among which $A B A$ is the candidate mediator.

\section{5 | Role of omics in improving drought stress resilience in rice}

Omics have emerged as a crucial tool to biotechnological and breeding approaches in the crop improvement missions in different crops including rice. Omics strategies have successively been used for identification of drought responsive genes, their expression controls regulatory network and the associated functional proteins and metabolic changes in plants. These studies may provide insights into the potential targets for rice improvement under drought conditions (Hamzelou et al., 2020). Deciphering the stress responsive circuit in rice plant during drought conditions can provide a clear understanding of how rice cultivars respond to drought stress and help in developing efficient drought-tolerant varieties to address food security raised by water scarcity.

\section{$2.6 \mid$ Genomics}

Genomics have emerged as an efficient bioinformatics platform to unravel the genetic basis of tolerance against drought stress in crop plants and the comprehensive data on candidate genes, alteration in their expression profiles during drought stress conditions. Modern and conventional breeding approaches for development of droughttolerant crop plants are not efficient until molecular mechanisms associated with stability of grain yield are not known in detail (Sinclair, 2011). The rice subspecies (Indica and Japonica) have been sequenced (Goff et al., 2002; Yu et al., 2002) providing plenty of genomics resources for enhancing drought tolerance. Analysing genomicsbased changes in large germplasm sets of rice may reveal certain breeding signatures that include key functional genes and loci associated with important agronomic traits are highly efficient and potential targets for rice improvement (Xie et al., 2015). Advancements in genetic techniques combined with genomics, breeding and precise phenotyping can thus be a reliable approach for deciphering putative genes, their expression control network, and metabolic pathways associated with drought tolerance that can be exploited for improvement of drought stress in rice (Sahebi et al., 2018). With advancement of next generation sequencing (NGS) technology, progress has been made in studying genetic diversity, population structure analysis, cluster analysis, gene diversity studies and identifying small genetic variations among the individuals (Islam et al., 2018). Extensive mapping of genome variability and population structure of rice may facilitate genome-wide association studies (GWAS) of complex traits, functional gene analysis and with phenotypic attributes-based cluster analysis may help in selection of donors for breeding purpose (Islam et al., 2018). However, limited progress in phenotyping tools have resulted in a phenotyping bottleneck limiting the genetic dissection of complex drought associated traits in rice (Cobb et al., 2013; Melandri et al., 2020). Genomics-assisted breeding approaches have proven advantageous for drought tolerance in rice by identification of stress associated loci which can be further subjected to breeding programmes (Figure 1). Plasticity in root traits of rice ameliorates drought tolerance as discussed earlier, thus deciphering their molecular and genetic mechanisms might add value to effective crop breeding efforts. In this context, three genomic loci for root architecture and a locus for both root architecture and grain yield were identified as the hotspots for traits associated with plasticity of root architecture using SNP markers (Sandhu et al., 2016). Studies involving combination of quantitative trait locii (QTLs) associated with drought stress responses aid in characterisation of physiological mechanisms associated with drought tolerance as observed in the lines derived from 
crossing IR64 $\times$ AdaySel varieties where all the possible QTL combinations of four QTLs i.e. $q D T Y_{2.2}, q D T Y_{4.1}, q D T Y_{9.1}$ and $q D T Y_{10.1}$ were used for characterising grain yield, harvest index, flowering, leaf water status, shoot growth, root architecture and its functional parameters such as water uptake, hydraulic conductivity (Henry et al., 2015). Dissection of drought QTLs using expressed sequence tags (ESTs) and the related candidate genes associated with drought stress has been done via functional genomics-based analysis of drought stress responses in rice by transcript mapping of 2095 unigene set together with 589 putative stress responsive genes that paves the way for molecular breeding pipeline (Markandeya et al., 2005). Transcriptome analysis of $\sim 21000$ genes of two phenotypically divergent rice cultivars (CT9993 and IR62266) and their transgressive segregants associated to changes in expression of drought related genes with QTLs for osmotic adjustments were reported (Hazen et al., 2005).

Genomics studies have also been exploited to identify and functionally characterise crucial genes associated with ABA-mediated drought signalling such as PYR/PYL/PP2C, SnRK to mediate drought tolerance (Xue et al., 2008; Tian et al., 2015). Computational genomics revealed 12 orthologs of $A B A$ receptors in rice (OsPYLs) that existed in various oligomeric states such as dimers, monomer-dimer equilibrium and inhibited the activity of diverse PP2Cs either in ABAdependent manner or ABA-independent manner (He et al., 2014). Moreover, a comparative genome-wide analysis of the PP2C family in rice and Arabidopsis revealed 80 and 78 genes, respectively, and characterised the identified genes on the basis of phylogeny in monocots and dicots, functional divergence, and regulatory mechanisms (Xue et al., 2008). Thus, genomics-based trait discovery, characterising the genetic basis of drought tolerance along with the identification of candidate genes may further be used as potential targets to assist in development of tolerant varieties and lend a new way for agriculture sustainability.

\subsection{Proteomics}

Plant responses to drought stress conditions is accompanied by changes in expression of various proteins (Wang et al., 2016) and thus, a proteomics approach is another efficient aid in identification and characterisation of the proteins that are altered in response to stress conditions and their role in drought tolerance. Technical advancements in proteomics in the past decade have facilitated the identification of several drought-responsive proteins in rice (Kim et al., 2014). Proteomes of different tissues including leaves, roots, spike, spikelet and seeds have been followed up to identify the drought stressresponsive proteins by both bottom-up and top-down proteomics approaches (Kim et al., 2014). Moreover, in order to get an insight into the drought-responsive proteins in different subcellular organelles, proteome analyses of the nucleus (Choudhary et al., 2009; Jaiswal et al., 2013), extracellular matrix (Pandey et al., 2010) and chloroplast (Gayen et al., 2019) were carried out by primarily utilising a gel-based proteomics approach except for chloroplast proteome analysis where an isobaric tag for relative and absolute quantitation (iTRAQ)-based quantitative approach was employed. Besides, an insight into the post-translational regulation of the drought-responsive proteins was also provided in rice leaves by a phosphoproteome analysis (Ke et al., 2009). The identified drought-responsive proteins have been mapped to various pathways such as ROS detoxification, primary and secondary metabolism, protein folding (chaperon activity), and stress/defence response. Proteome analysis of two rice genotypes including IR64 (drought-sensitive) and Moroberekan (droughtresistant) revealed the presence of beta-expansin, actin-binding protein, glyceraldehyde-3-phosphate dehydrogenase and pectinesterase inhibitor domain-containing protein as the product of droughtinduced proteins, without affecting starch accumulation in the drought-tolerant genotype while no such effects were observed in sensitive genotype (Liu and Bennett, 2011). Conversely, the expression of drought-induced glycoprotein Os08g12160 identified was much higher in drought-sensitive variety. Together, all of these studies have led to the identification of an array of protein candidates to be targeted for the development of rice cultivars with enhanced drought tolerance.

Comparative proteomics is the foundation stone for the analysis of drought stress and other abiotic stresses in plants (Gupta et al., 2015a, 2015b). Two-dimensional gel electrophoresis (2-DGE) remained the method of choice for comparative proteome analysis for the identification of stress-responsive proteins in rice, however, efforts have also been put to employ the high-through shotgun proteomics including label-free quantification (Meng et al., 2018, 2019) and/or tandem mass tags/isobaric tag for relative and absolute quantitation (TMT/iTRAQ)-based quantification (Gupta et al., 2019). Interestingly, several studies based on root proteome analysis (Mirzaei et al., 2012a) and extracellular matrix proteome analysis (Pandey et al., 2010) have shown increased abundances of HSPs and other chaperones in rice during drought stress suggesting their roles in drought stress tolerance (Shu et al., 2011). In a comparative proteome profiling of eight rice diverse genotypes (including both japonica and indica sp.) identification of an 18.6 kDa class III small HSP (HSP18.6) as a drought-induced protein along with four isoforms of LEA proteins were detected in all cultivars (Hamzelou et al., 2020). N22 genotype that is highly tolerant to drought stress displayed maximum accumulation of HSP18.6 and four LEA proteins confirming the pivotal roles of these proteins in drought stress tolerance (Hamzelou et al., 2020). Moreover, drought stress-induced elevation in different isoforms of LEA proteins including group 6 LEA protein, LEA type-1 protein, LEA protein and putative HSP further confirms their role in drought tolerance in rice (Muthurajan et al., 2011). HSPs are the stress responsive proteins that are induced as an acclimation response of plants to dehydration stress to sustain their growth and survival (UI Haq et al., 2019). Furthermore, a combination of label-free and TMT-based quantitative proteome analysis was used for comparative proteome analysis of two rice cultivars including Nipponbare (drought-sensitive) and IAC1131 (drought tolerant) (Wu et al., 2016a). Interestingly, both the cultivars showed increased abundances of chaperone protein ClpB1, 17.9 kDa class I HSP, (Hsp17.9) and 18.6 kDa class III HSP (Hsp18.6) upon extreme drought stress, suggesting that both the 
cultivars employ a similar mechanism to combat the drought stress Putting together, all of these studies collectively suggest a key role of HSPs and other chaperonins in drought stress tolerance.

There are several proteins associated with the biosynthesis of plant hormones, their signalling pathways and drought tolerance (Rabello et al., 2014). Owing to the low abundance of the hormoneresponsive proteins, it is relatively difficult to identify the proteins related to the hormone biosynthesis and signalling using a gel-based proteomics approach. Therefore, a much less number of drought stress modulated hormone-responsive proteins have been identified so far. Yet, this limited information suggested a positive regulation of $A B A$ and a negative regulation of $J A$ in drought stress tolerance in rice (Dhakarey et al., 2017). However, a contradictory positive regulation of JA in drought stress signalling has also been depicted (Wu et al., 2016b). Further experimentations are required to assign the clear roles of JA in drought stress tolerance in rice. Moreover, an $\mathrm{ABA}$ and stress-inducible protein was also found to be 6.2-fold induced in response to drought stress in rice leaves (Rabello et al., 2014). Increased abundance of these proteins suggested a positive regulation of ABA. Employing a label-free quantitative proteomics analysis, the proteomes of transgenic and wild-type rice were compared which led to the identification of OsPP2C as a downregulated protein in transgenic rice (Shi et al., 2018). As OsPP2C is a negative regulator of ABA-signalling in plants (Gosti et al., 1999; Gupta et al., 2018), downregulation of this protein in response to drought stress suggests positive regulation of $A B A$ in drought stress tolerance in rice (Gupta et al., 2019).

In order to elucidate the effect of intermittent drought stress on rice leaves, Rabello et al. (2014) utilised a 2-DGE-based proteomic approach. This study led to the identification of 15 differential proteins of which seven showed increased abundance including an APX and a GSH-dependent dehydroascorbate reductase 1 (Rabello et al., 2014). The proteins related to energy metabolism and anabolic processes were increased in response to drought stress (Shu et al., 2011; Jaiswal et al., 2013). The PR-proteins (pathogenesis-related proteins) may play a crucial role in mediating plant growth and metabolism and enhance drought tolerance during drought stress conditions (Lee et al., 2008). Shotgun proteome analysis showed the accumulation of six PR-proteins in rice roots upon drought stress (Mirzaei et al., 2012b). Additionally, a similar study also led to the identification of six chitinases as droughtinduced proteins in the rice roots that mediates root architecture and defence system of the plant to ensure its growth, development and yield during drought condition (Mirzaei et al., 2012b). Conclusively, proteome analysis has emerged as an efficient tool to further explore the proteins associated with drought responsiveness in rice that may further permit the identification of genes and novel response pathways associated with drought tolerance.

\section{8 | Metabolomics}

Metabolomics is an effective tool with multifarious approaches for unravelling and characterising stress responsive metabolites in plants.
Efficiency of drought tolerance requires a completely functional metabolism in crops during drought stress (Hu and Xiong, 2014). Drought stress affects various metabolic pathways of rice including primary and secondary metabolism, energy metabolism, redox homeostasis and signalling proteins. Photosynthesis being the most important drought acclimation response subsequently decreases during drought conditions (Saibo et al., 2009). Drought tolerance mechanisms such as ROS scavenging, osmolytes (proline, soluble sugars, polyamines) accumulation, alteration in levels of protective metabolites and biomolecules such as proteins and lipids are involved in maintaining optimal growth during drought conditions (Serraj and Sinclair, 2002; Saibo et al., 2009). Thus, insights to metabolomics may provide potential targets that regulate drought tolerance. Metabolomics together with gene expression profiling may be used in deciphering the signature markers or potential targets that are associated with important traits contributing to drought tolerance (Degenkolbe et al., 2013).

In rice, metabolomic studies accompanied by transcriptomics looking at photosynthetic regulation and drought tolerance revealed the presence of 69 differential metabolites (DMs) and 4059 differentially expressed genes (DEGs) within IRAT109 (drought-intolerant) and, 47 DMs and 2677 DEGs in IAC1246 (drought tolerant) cultivars, respectively (Ma et al., 2016). 6 DMs were correlated with osmotic potential and antioxidant capacity while the performance of photosynthetic DEGs consistently upregulated certain metabolites such as 4-hydroxycinnamic acid and ferulic acid during early drought stages in IAC1246 suggesting a role for metabolites in maintaining photosynthesis during drought conditions via osmotic adjustments and ROS scavenging (Ma et al., 2016). Metabolic pathways exhibited significant genotype and environment $(\mathrm{G} \times \mathrm{E})$ interaction in an expressional profiling study and identified metabolites that may be exploited for modulating drought tolerance in wide range of rice germplasms. Metabolic analysis in 21 rice cultivars from both indica and japonica exhibited a negative correlation between metabolite levels (like asparagine, glutamine, glutamate, serine, glycine, threonine, erythronic acid, galactonic acid and threonic acid) and plant performance (such as stay green trait, water use efficiency, total fresh and dry weight, photosynthetic yield and shoot fresh and dry weight) under drought condition while a positive correlation exists between expression levels and plants performance for 28 genes out of a total of 46 candidate genes (Degenkolbe et al., 2013).

Constitutive metabolic markers associated with the reproductive traits during combined effect of heat and drought stress in rice have also been identified via metabolome and transcriptome analysis highlighting differential metabolic profiles across anthers and pistils (Li et al., 2015). Additionally, sugar metabolism emerged as the most important metabolic and transcriptional component during drought and heat stress in the heat-tolerant variety (N22). It was also observed that, expression of cell wall invertase (INV4) and sugar transporter (MST8) enhanced in tolerant N22 variety, while the expression of the CARBON STARVED ANTHERS (CSA) gene was enhanced in the susceptible variety (Moroberekan) in response to combined drought and heat conditions (Li et al., 2015). Further, an integrated metabolomic, 
proteomic and physiological analysis (Xiong et al., 2019a, 2019b) suggested a decrease in soluble sugar levels and net photosynthetic rate upon drought stress while the activities of ROS detoxifying enzymes increased significantly. Metabolome and proteome results further supported the observation of modulation of ROS metabolism by the abrupt drought-flood alternation stress in rice (Xiong et al., 2019a, 2019b). Thus, a significant analysis of the drought responsive metabolome in tolerant and susceptible cultivars may unravel the essential fundamentals pertaining to drought stress adaptation.

\section{9 | Plant genome editing and improving drought- tolerance in rice}

The advent of genome editing technologies overcomes the traditional breeding method limitations, and aids in achieving the accelerated crop breeding by enhancing the genetic gains. Genome editing tools utilises the use of site-specific nucleases (SSNs) such as transcriptional activator-like effector nucleases (TALENs), zinc finger nucleases (ZFNs) and most recent one clustered regularly interspaced short palindromic repeats (CRISPR)/CRISPR-associated protein-9 (Cas9) systems, which enable the precise modifications of target genes at a desirable location in the predefined manner (Komor et al., 2016; Ansari et al., 2020). Among different SSNs, CRISPR has overtaken others due to its specificity and precision that allows targeted genome editing (Gao et al., 2017). All SSNs breaks the target DNA sequence at specific sites and utilises the plant's natural DNA repair mechanism to repair the DNA double-strand breaks (DSBs) through either homologous recombination (HR) or non-homologous end joining (NHEJ). The NHEJ repair pathway is error-prone, thus resulting in the intended sequence alterations ranging from frame shift mutations, point mutation to large insertions or deletions (INDELs) at predefined sites in the target genomes. This tool has been used successfully in most plants including rice due to its high precision, simplicity and adaptability. The homology-directed repair (HDR) pathway requires a donor template or foreign DNA for knock-ins at the site of the DNA break (Chang et al., 2015). The CRISPR/Cas system is an efficient, user friendly and accurate genome editing tool developed in past few years (Shan et al., 2013). Presently, this tool has been widely applied in major crops such as sorghum (Li et al., 2018a), maize (Svitashev et al., 2015; Zhu et al., 2016) and barley (Gasparis et al., 2018). Tolerance against abiotic stress is a complex phenomenon involving an array of various tolerance mechanisms regulated by multistep gene expression networks to maintain cellular homeostasis (Mickelbart et al., 2015). The CRISPR/Cas9 system is one of the efficient tools which can be adopted immediately to target the crucial candidate genes and metabolic pathways regulating abiotic stress tolerance to develop abiotic stress-tolerant crops including drought stress. But to date, only a few studies have been directed at drought stress improvement in rice using genome editing tools. The ERF family has been explored in rice for studies of drought tolerance. OsERF109 targeting helped developing water stress tolerance (Mishra and Zhao, 2018). It is previously known that the OsNAC14 gene confers drought stress tolerance in rice, and this was further confirmed using the CRISPR-Cas9 system (Shim et al., 2018). Mutants of SAPK2, a gene that primarily mediates ABA signalling during drought stress were produced using CRISPRCas9 and showed a high sensitivity to drought stress and ROS accumulation implying the potential role of SAPK2 in drought tolerance in rice (Lou et al., 2017). Similarly, knockout of OsNAC006 TF through CRISPR/Cas9 tool resulted in drought and heat sensitivity in rice (Wang et al., 2020b). Genome editing has turned up as a robust approach for targeted rice improvement under drought stress conditions by specifically altering candidate genes for drought responses across the plant genome. Thus, harnessing genome editing for crop improvement and stress tolerance is an application platform to enhance the crop adaptability and increase its economic value during drought conditions.

\subsection{0 | Development of rice transgenics for drought stress tolerance}

Trait improvement through traditional breeding is labour-intensive, cost-intensive and time-consuming process for achieving higher yield under drought conditions. Contrary to breeding and marker-assisted selection approaches, manipulation of gene expression using genetic engineering seems to be more attractive and immediate way for developing stress-tolerant crops (Ahmar et al., 2020). Genetic engineering has thus been intensively explored for two decades to improve plant tolerance to abiotic stresses and other traits (Cui et al., 2018, 2020). Till now, several studies exploiting genetic engineering approaches have been done to produce drought-tolerant rice varieties in order to promote sustainable agriculture (Du et al., 2010; Ganguly et al., 2020). Overexpression of the ARGININE DECARBOXYL$A S E(A D C)$ gene from oat in transgenic rice under the control of the CaMV35S promoter reduces chlorophyll loss, increases the accumulation of polyamines such as putrescine, that mitigates stress-induced oxidative damage by inducing the activity antioxidant enzymes, during drought conditions (Capell et al., 1998). While overexpression of the $A D C$ gene from Datura stramonium in transgenic rice under the control of the Ubi-1 promoter ameliorated drought tolerance by enhancing putrescine and spermidine levels (Li et al., 2011). Genetic engineering has been done to produce a transgenic rice variety with improved drought tolerance by overexpressing OsSRO1c gene under the control of Ubi1 resulting in reduced enhanced stomatal closure, prevented water loss and mitigated ROS accumulation (You et al., 2013).

Alteration in expression of several genes such as OsSGL (Cui et al., 2016), OsGRXS17 (Hu et al., 2017), OsPM1 (Yao et al., 2018) and OsSCE3 (Joo et al., 2019a) play a crucial role in drought tolerance by modulating drought associated traits such as accumulation of osmolytes, expression of stress-responsive genes, ROS accumulation, stomatal closure and modification of root systems. Recently, the drought stress-tolerant transgenic rice variety Pusa Sugandhi 2 was developed by individual overexpression of OsRab16A and AtDREB1A genes involved in $A B A$ dependent and $A B A$ independent pathway of 
drought tolerance, respectively (Ganguly et al., 2020). Both sets of transgenic plants exhibited drought tolerance by reducing inhibition of root and shoot length, increasing leaf relative water content with enhanced CAT activity to reduce oxidative damage. The overexpression of OsRab16A and AtDREB1A also resulted in increased chlorophyll content, proline content, higher filled grain, spikelet fertility and grain yield under stressed conditions (Ganguly et al., 2020). Overexpression of a multi stress-tolerant gene from rice, that is, the ornithine $\delta$-aminotransferase (OsOAT) gene significantly increased drought and osmotic stress tolerance (You et al., 2012). The OsOAT overexpressing transgenic rice varieties resulted in reduced wilting, delayed senescence, high seed setting rates, enhanced antioxidant enzymes, increased activity of ornithine $\delta$-aminotransferase and high proline levels during drought conditions (You et al., 2012). Likewise, several studies have been conducted employing genetic engineering for the development of transgenic rice variety with improved drought tolerance by enhancing drought associated traits such as ROS scavenging, stomatal closure, improved phenotypes and increased yield (Abreu et al., 2018; Qin et al., 2020). In rice, the OsGF14b gene of the14-3-3 protein family was functionally characterised (by using both overexpression and RNAi lines) and analysis reveals that it is involved in the drought resistance in rice plants, partially in an ABAdependent manner (Liu et al., 2019). Overexpression of the rice ANNEXIN (OsANN3) gene showed improved drought stress tolerance with longer root length and more stomatal closure for less water loss by regulating $\mathrm{ABA}$-dependent stress response pathways (Li et al., 2019a). Transgenic rice plants overexpressing Oryza sativa DROUGHT AND SALT STRESS RESPONSE-1 (OsDSSR1) exhibit enhanced drought stress with the accumulation of compatible osmolytes, such as free proline and soluble sugars (Cui et al., 2018). Transporters such as $\mathrm{K}$ and nitrate transporter proteins also play a major role in achieving stress tolerance. The overexpression of rice high-affinity nitrate transporter partner protein (OsNAR2.1) plays an essential role in increasing grain yield under drought stress conditions (Chen et al., 2019). OsHAK1, a high-affinity $\mathrm{K}$ transporter-mediated amelioration of drought tolerance in rice at the reproductive stage and can be considered to be used in introgression strategies in the rice breeding programmes (Chen et al., 2017). Approaches based on genetic and metabolic engineering in rice have prominently revolutionised the researches based on nutrition enhancement by optimising the expression of related genes associated with quality traits for crop improvement (Ahmad et al., 2016). In rice, OsAKT1 selectively alters $\mathrm{K}^{+}$uptake with its expression localised in the root cortex and its overexpression enhances $\mathrm{K}^{+}$uptake in rice in both drought and $\mathrm{K}^{+}$deficient conditions (Ahmad et al., 2016). Similar effects of a high affinity $\mathrm{K}^{+}$transporter, OsHAK1 on $\mathrm{K}^{+}$acquisition and distribution under drought stress has been revealed via knockout mutants that show shunted root and shoot growth, oxidative damage with the poor architecture of the root system resulting in lower nutrient uptake and translocation in rice (Chen et al., 2017). The overexpression of OsHAK1 enhances drought tolerance by reducing lipid peroxidation, increasing antioxidant enzyme activities (such as POD and CAT) and proline levels, improving other physiological parameters such as photosynthesis, and upregulating the genes associated with $\mathrm{K}$ homeostasis (OsTPKb and OsAKT1; Chen et al., 2017). In rice, proline accumulation-mediated expression of high affinity $\mathrm{K}^{+}$transporter suggests the interlinking between osmolyte accumulation and nutritional status during drought stress conditions (Chen et al., 2017). Various similar molecular insights are being investigated to improve the nutritional status of rice plants with efficient productivity to meet the global demand for agriculture sustainability.

Genetic engineering has also been employed to some LEA genes within rice resulting in cultivars with improved drought stress tolerance (Babu et al., 2004; Duan and Cai, 2012). Controlled expression of barley HVA1 in transgenic basmati rice under the control of the Actin1 promoter significantly contributes to improved drought and salinity tolerance in terms of ion leakage, cell integrity and growth of the plant (Rohila et al., 2002). Overexpression of a LEA gene, OsLEA3-1 delays leaf wilting (that may lead to enhanced spikelet fertility), enhances grain yield and improves drought resistance in rice (Xiao et al., 2007). Also, LEA genes such as OsLEA3-2 of rice (a monocot), when overexpressed in dicot such as Arabidopsis, significantly induce drought stress tolerance in terms of strong growth performance from flowering to seed set stage, enhanced survival ratio and increased grain per spikelet (Duan and Cai, 2012). Receptor-like kinases (RLKs) such as OsESG1 regulates root crown development in rice and mediated drought tolerance by early crown root development that influences seedling growth and overall plant survivability during drought (Pan et al., 2020). Some NAD kinases have also shown their involvement in drought stress tolerance in rice. For instance, transgenic lines overexpressing OsNADK1 shows enhanced drought stress tolerance in rice in terms of maintained intracellular redox homeostasis, enhanced water retention ability, reduced water loss and enhanced levels of osmolytes such as proline (Wang et al., 2020c). Transgenic rice overexpressing AtGOLS2 has been developed that shows a significant increase in biomass, a higher number of panicles, reduced leaf rolling, enhanced drought recovery, early flowering, and ultimately enhanced grain yield and grain fertility under drought stress (Selvaraj et al., 2017). Transgenic rice developed using hormonal receptors also been shown to enhance drought stress tolerance in rice. Overexpression of the $A B A$ receptor protein OsPYL10 in transgenic rice enhanced $A B A$ accumulation, increased relative water content and grain yield, improved membrane stability index, high chlorophyll content and a lower amount of malondialdehyde (MDA) and $\mathrm{H}_{2} \mathrm{O}_{2}$ that ultimately ameliorated drought and cold stress tolerance in the indica rice variety (Verma et al., 2019). All the identified genes can be used as an efficient molecular tool to mitigate grain yield losses in rice despite genetic variations under drought stress. OsIAA6 overexpressed in transgenic rice exhibited increased auxin biosynthesis genes that controlled drought tolerance and regulated tiller outgrowth (Jung et al., 2015). Auxin-induced drought tolerance in rice was mediated via the overexpression of OsPIN3t, an auxin efflux carrier gene involved in auxin transport (Zhang et al., 2012). In rice, various regulatory proteins also mediate resistance or tolerance against drought conditions (Lu et al., 2009; Zhang et al., 2010). Several regulatory proteins associated with drought stress tolerance have been identified in 
rice using genomic tools and their expression has been manipulated by genetic engineering to develop drought-tolerant transgenic rice varieties. For instance, transgenic rice generated by overexpression of OsSDIR1 gene showed reduced leaf rolling induced by drought conditions, enhanced chlorophyll content, increased tiller numbers, green and expanded leaves, stomatal closure, increased seed weight and overall a better survival rate (Gao et al., 2011). Overexpression of OsRDCP1, a RING domain-containing protein in rice results in reduced drought associated symptoms in transgenic lines with reduced desiccation suggesting the role of OsRDCP1 in drought stress tolerance in rice (Bae et al., 2011). Thus, altering the expression of genes and metabolic pathways associated with plant responses to drought stress using genetic engineering has been emerging as a way to produce drought-tolerant varieties and crop improvement.

\subsection{1 | Transcription factors involved in drought stress tolerance}

One important group of regulatory proteins that mediate drought stress tolerance in rice are TFs or trans-acting elements. TFs regulate the expression of drought stress associated genes. Plants have developed an efficient acclimation mechanism to cope with drought conditions by manipulating the transcriptional control networks via drought responsive TFs families such as NAC, AP2/ERF, bZIP and MYB (Nakashima et al., 2007; Jung et al., 2017). Many TFs have been identified in rice that are associated with drought tolerance traits and studies have shown that overexpressing drought responsive TFs may induce stress tolerance and may be used to develop drought-tolerant rice varieties (Yang et al., 2012b; Jung et al., 2017) (Table S1). Additionally, TFs have been identified that mediate drought escape strategies adapted by rice plants to protect themselves from droughtinduced damages (Table 2). For instance, a WUSCHEL Homeobox TF, WOX13 helps rice plants to cope with adversities of drought stress by inducing the escape strategy early flowering that helps the plant complete its life cycle before the emergence of severe stress conditions that may lead to death (Minh-Thu et al., 2018). Overexpression of OsNAC14 induced drought stress tolerance in rice at the vegetative stage with high panicle number, enhanced grain filling rate, biosynthesis of strigolactones and induction of stress responsive genes (Jeong et al., 2013). Similarly, TFs OsbZIP71 (Liu et al., 2014), OsERF71 (Lee et al., 2016), OsERF48 (Jung et al., 2017) and OsMYB2 (Yang et al., 2012b) have shown to enhance tolerance against drought stress in rice.

Basic leucine zipper (bZIP) TFs are important regulatory elements that control drought stress responses in plants. In rice, overexpression of OsbZIP62 (a novel bZIP TF involved in ABA signalling) enhances tolerance against drought stress at the vegetative stage. The overexpression lines show low wilting, less ROS accumulation, enhanced chlorophyll content as compared to wild-type (WT) plants, while osbzip62 knockout mutant lines exhibited more intensifying drought symptoms compared to WT, with worsen leaf rolling, high wilting, high oxidative stress and low survival rates (Yang et al., 2019). Likewise, in transgenic rice overexpressing OsbZIP42 resulted in enhanced expression of LEA3 and Rab16 (ABA-responsive genes) and the seedlings that were hypersensitive towards $A B A$ exhibited low germination rate, reduced root and shoot growth in the presence of ABA (Joo et al., 2019b). OsABF1, another member of bZIP family, confers drought tolerance in transgenic rice by mediating drought-induced upregulation of COR413-TM1 gene (Zhang et al., 2017).

$\mathrm{C}_{2} \mathrm{H}_{2}$-type zinc finger proteins are considered as one of the largest family of TFs that regulate drought stress tolerance in rice (Agarwal et al., 2007). Unlike other members of the $\mathrm{C}_{2} \mathrm{H}_{2}$-type zinc finger proteins family that mediates drought tolerance with negative effects on plant growth, DROUGHT-RESPONSIVE ZINC FINGER PROTEIN 1 (OsDRZ1) has been identified as a positive regulator of drought tolerance in rice that mitigates drought stress adversities and maintains optimal plant growth (Yuan et al., 2018). Transgenic rice with overexpressed OsDRZ1 that positively regulated plants optimal growth under drought stress tolerance in rice showed high accumulation of osmolytes, enhanced activity of antioxidants enzymes, reduced ROS production, increased stomatal closure, reduced water loss, higher survival rates and better growth performance (Yuan et al., 2018).

NAC is an important TF that belong to the largest plant-specific TF family (Shao et al., 2015). Several TFs from the NAC family have shown their role in mediating drought stress in plants by regulating the expression of stress responsive genes that mediate plant tolerance mechanisms associated with drought tolerance (Fang et al., 2015). For example, in rice SNAC1 confers drought tolerance by mediating the expression of OsPP18 gene that is independent of ABA (You et al., 2014). Expression control of several other NAC TFs such as OsNAC10 (Jeong et al., 2010), OsNAC9 (Redillas et al., 2012), OsNAC5 (Song et al., 2011), OsNAC6 (Nakashima et al., 2007) has also shown to mediate drought tolerance in rice. Apart from positive regulators for drought stress tolerance, there are several studies highlighting TFs that are negative regulators of drought tolerance in rice (Huang et al., 2016). For instance, overexpression of ET-responsive element binding protein (OsEBP89) that belongs to AP2/ERF subfamily of TFs negatively regulates drought stress tolerance in rice by increasing drought stress related damages in the plant such hyperaccumulation of ROS, reduced antioxidant enzymes (Zhang et al., 2020b). Similarly, TFs including OsERF109 (Yu et al., 2017), OsETOL1 (Du et al., 2014), OsDERF1 (Wan et al., 2011) and OsERF3 (Zhang et al., 2013) have also been identified as negative regulators of drought tolerance. Along with this, there is a wide range of TFs from the MYB family such as OsMYB6 (Tang et al., 2019) that are involved in mediating drought stress tolerance in rice. The DREB genes are crucial transcription regulators that are known to play a crucial role in drought stress tolerance. For instance, OsDREB1 when overexpressed in transgenic rice enhances drought tolerance in rice and exhibits accumulation of soluble sugars and osmolytes such as proline, enhanced survival and growth of the plant (Ito et al., 2006). Furthermore, identification and characterisation of more TFs associated with drought stress tolerance may provide better insights for the expression network of drought 


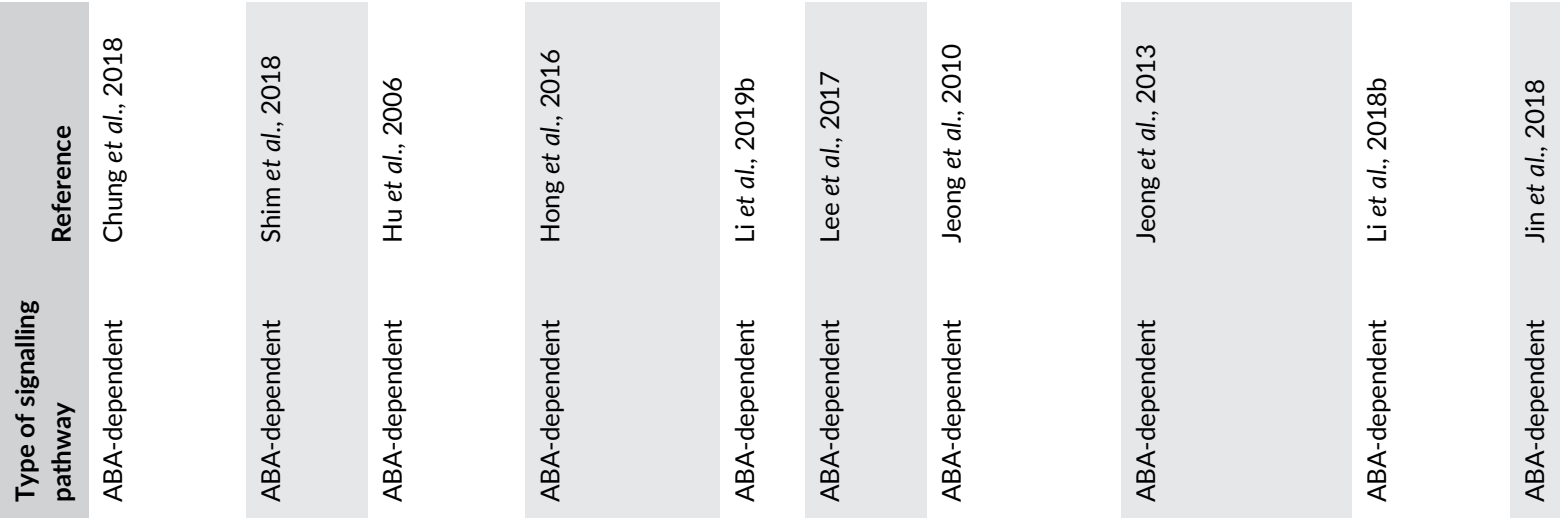
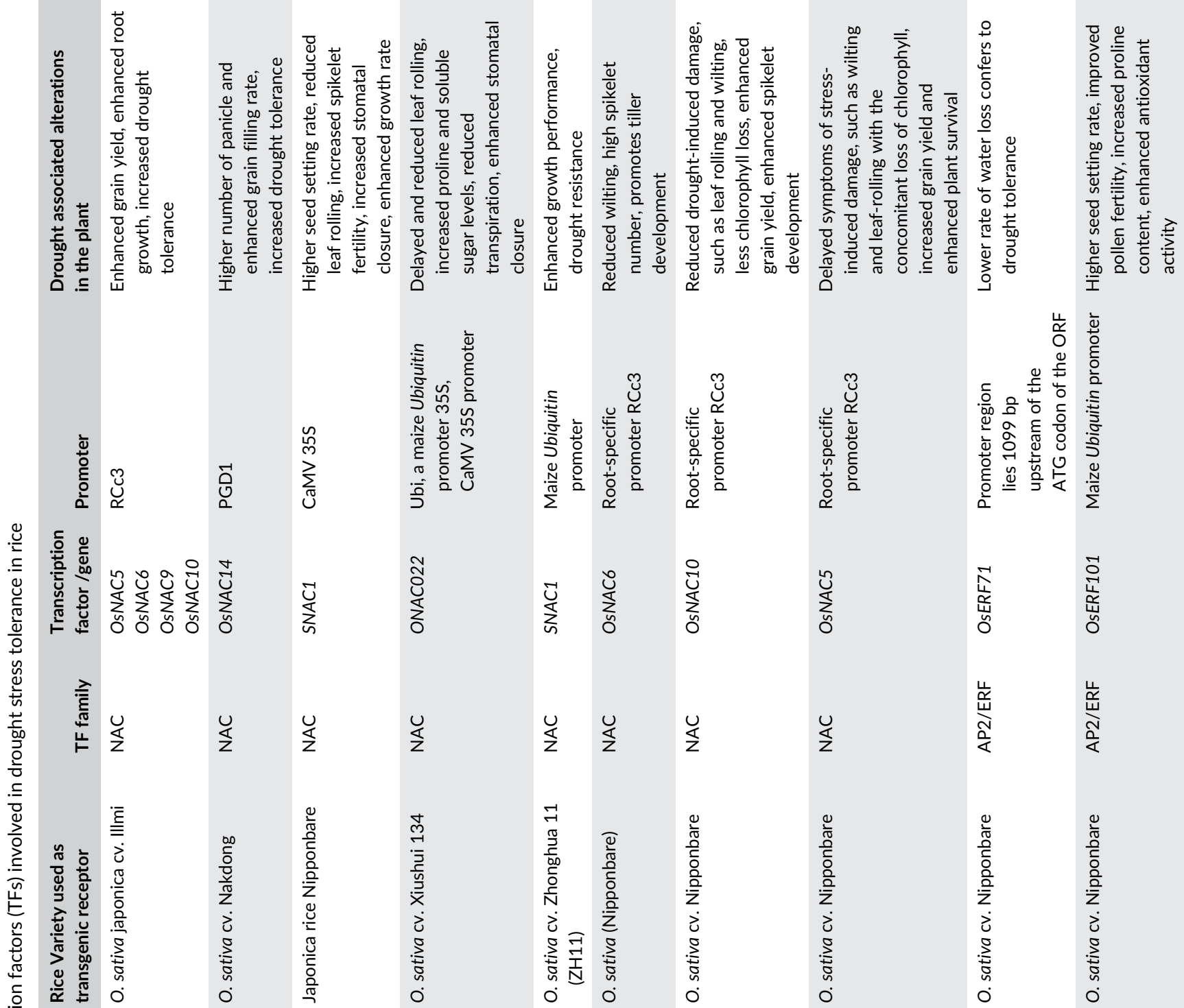

$\frac{u}{z} \quad \frac{y}{z}$

$\begin{array}{llll}\frac{y}{z} & \frac{U}{z} & \frac{y}{z} & \frac{y}{z}\end{array}$

y

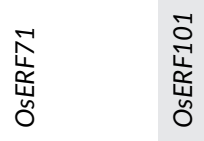

$\stackrel{2}{2}$
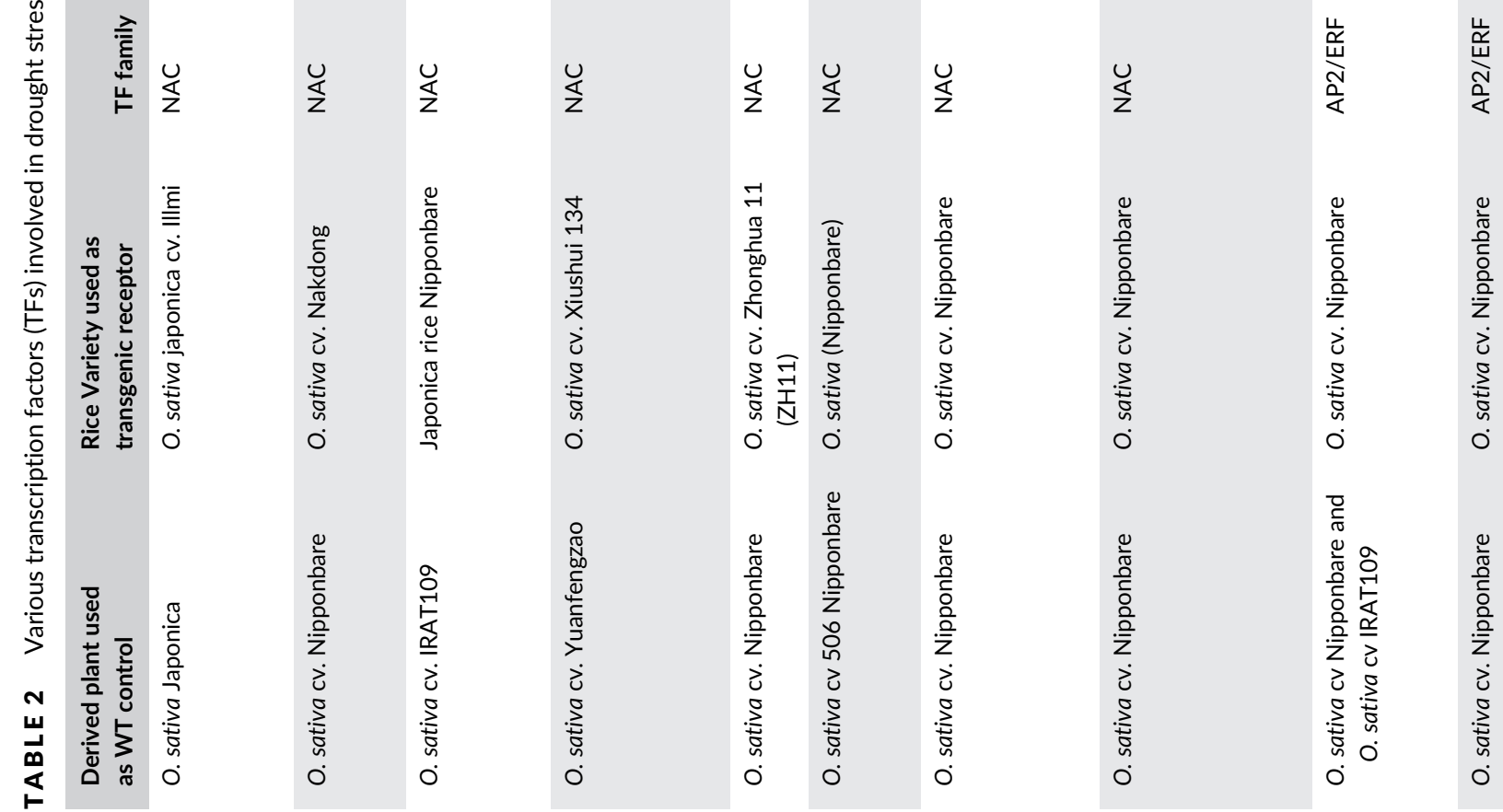


\begin{tabular}{|c|c|c|c|c|c|c|c|c|c|c|}
\hline 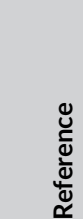 & 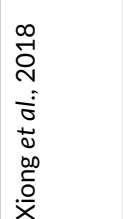 & 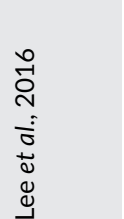 & 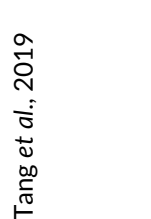 & 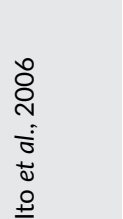 & 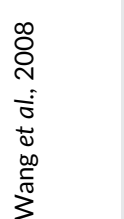 & 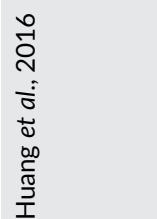 & 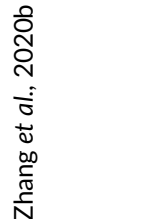 & 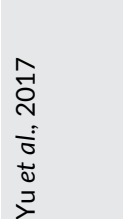 & 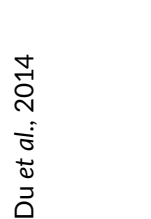 & $\begin{array}{l}\text { ने } \\
\text { N } \\
\dot{0} \\
\dot{0} \\
\overrightarrow{0} \\
\overrightarrow{1}\end{array}$ \\
\hline 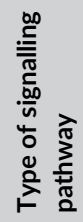 & 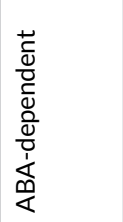 & 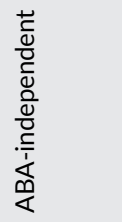 & 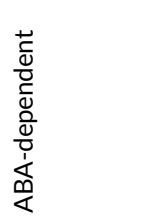 & 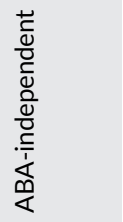 & 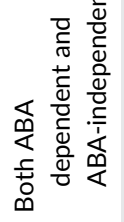 & 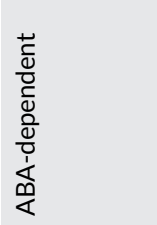 & 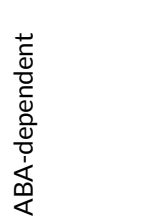 & 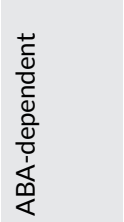 & 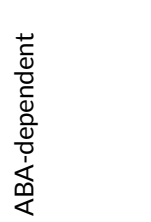 & 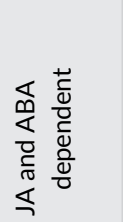 \\
\hline 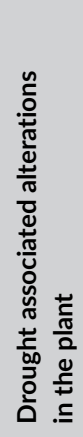 & 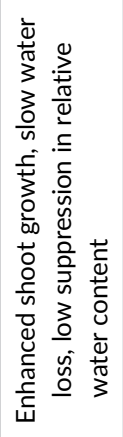 & 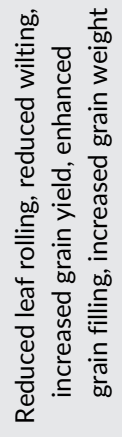 & 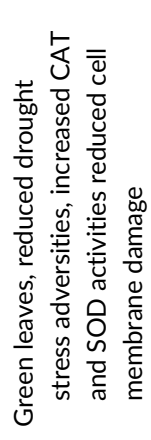 & 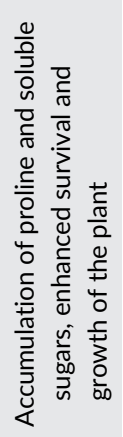 & 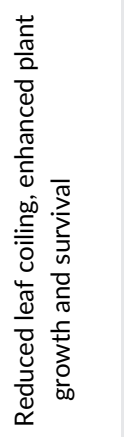 & 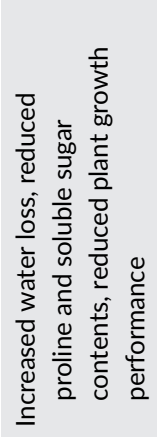 & 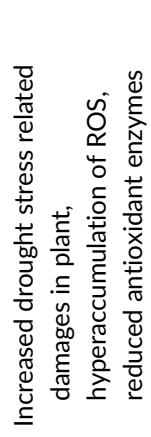 & 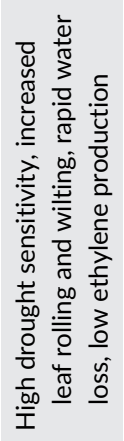 & 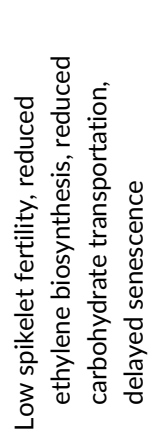 & 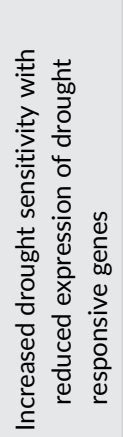 \\
\hline $\begin{array}{l}\bar{\nu} \\
\stackrel{\text { on }}{0} \\
\stackrel{0}{0}\end{array}$ & 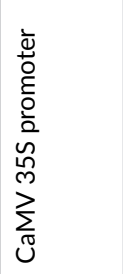 & 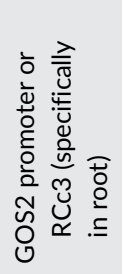 & 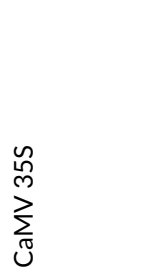 & 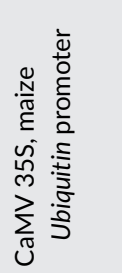 & 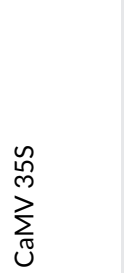 & 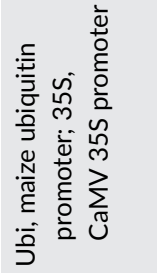 & 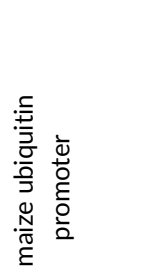 & 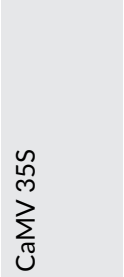 & 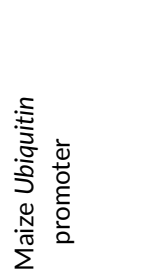 & 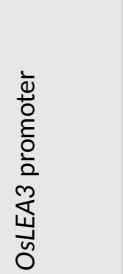 \\
\hline 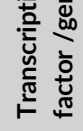 & 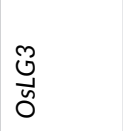 & $\begin{array}{l}\stackrel{-1}{u} \\
\text { 岕 } \\
\text { on }\end{array}$ & $\sum_{\substack{\infty \\
0}}^{\infty}$ & 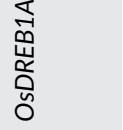 & 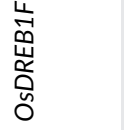 & $\overbrace{0}^{n}$ & 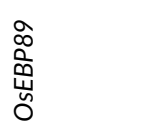 & $\begin{array}{l}\text { Oे } \\
\text { 竞 } \\
\text { 岀 } \\
0\end{array}$ & 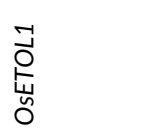 & $\frac{\vec{N}}{\hat{\Delta}}$ \\
\hline 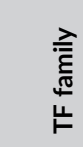 & 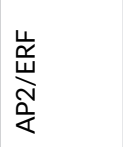 & 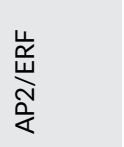 & $\sum_{\Sigma}^{\infty}$ & 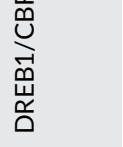 & 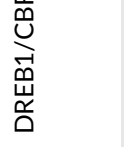 & U̦ & 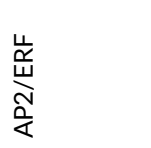 & 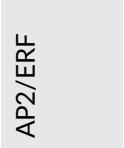 & 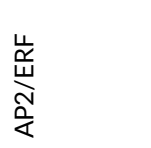 & $\mathbb{1}$ \\
\hline 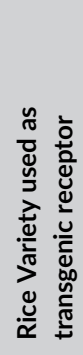 & 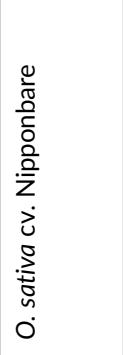 & 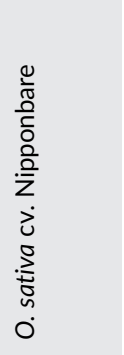 & 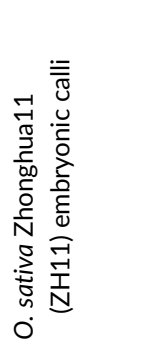 & 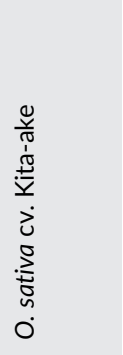 & 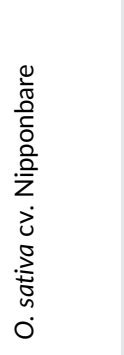 & 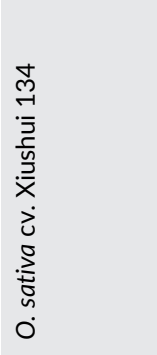 & 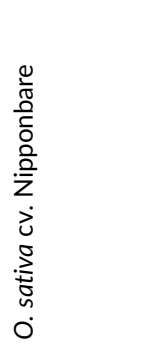 & 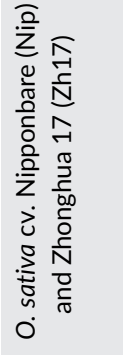 & 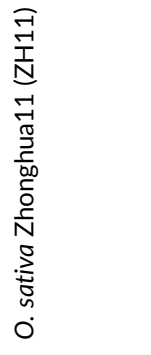 & 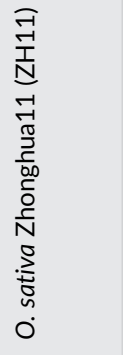 \\
\hline 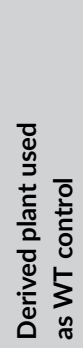 & 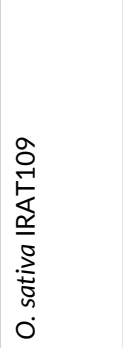 & 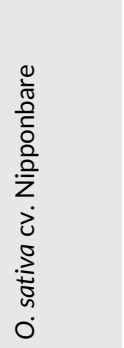 & 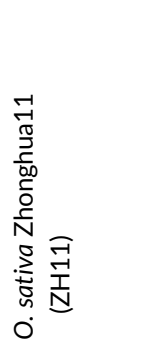 & 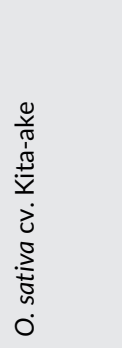 & 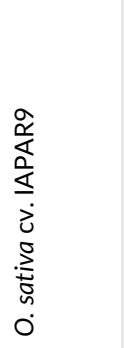 & 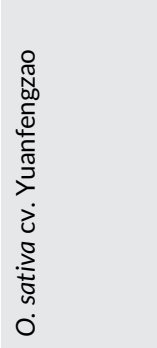 & 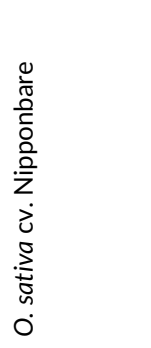 & 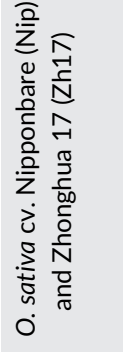 & 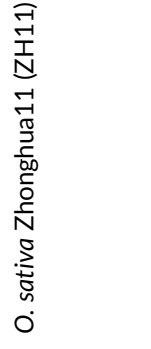 & 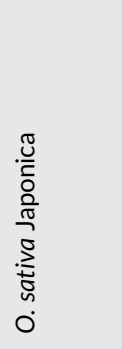 \\
\hline
\end{tabular}


related genes and possibly aid in the production of better tolerant varieties.

\subsection{2 | Efforts in molecular breeding and drought- tolerant rice}

Naturally distributed rice cultivars over different geographical areas are the source of genetic variability that can be highly efficient for the breeders to develop different cultivars with desirable traits using natural genetic resources (Patra et al., 2016). Prominent producers of rice such as Asia and Africa are usually rainfed but conditions such as abnormal rainfall patterns and droughts adversely affect rice growth and development prominently at the reproductive stage. Therefore, exploring the availability of the genetic resources for drought tolerance is of paramount importance. Genetic variability in the rice germplasm needs to be deciphered for the identification of drought associated attributes and to assist in designing breeding strategies for the production of drought-tolerant cultivars.

Plant breeding approaches have significantly contributed to improving rice drought tolerance (Sandhu and Kumar, 2017). Moreover, the development of introgression lines with QTLs associated with drought stress using molecular and genomics-assisted breeding is needed for improving drought tolerance and grain yield. Drought tolerance in rice is a complex trait and thus needs a multifaceted breeding approach that also includes marker-assisted breeding (MAB) to enhance drought tolerance (Hao and Lin, 2010). The rice cultivars with high drought tolerance are the most promising source of genes that can be used in the development of drought tolerant or resistant varieties with appreciable nutritional value and yield. Drought tolerance research on rice are being extensively carried out at the International Rice Research Institute (IRRI), Philippines over decades, using traditional drought-tolerant donors and modern high-yielding varieties for mapping population development in controlled and drought conditions, and to identify major QTLs associated with grain yield under drought stress ( $q D T Y s$ ) (Swamy and Kumar, 2012). For instance, $q D T Y_{1.1}$ (Vikram et al., 2011), qDTY ${ }_{9.1}$ (Swamy et al., 2013), $q D T Y_{12.1}$ (Bernier et al., 2007), qDTY 2.1 (Venuprasad et al., 2009), are some of the major and highly effective QTLs for grain yield under drought conditions in rice. During drought stress, the QTL $q D T Y_{12.1}$ (Bernier et al., 2007) mediates two major physiological effects including enhanced growth in lateral roots and increased transpiration efficiency, and appears as a combinational trait in rice (Henry et al., 2014). The list of other major yield QTLs identified by yield as a selection criterion is summarised (Table S2).

Breeding approaches have facilitated the development of droughttolerant cultivars with a chance of obtaining segregants having precise combinations of traits that could meet local conditions and farmer's requirements. Consistent efforts in assisting the development of drought resilient cultivars by introgression of the identified $q D T Y_{s}$ into drought susceptible varieties through MAB are being made to help farmers with increased yield and productivity irrespective of unfavourable environmental cues especially in Asian countries (Sandhu et al., 2019; Vinod et al., 2019). In Nepal, drought-tolerant rice cultivars have been released after introgression of $q D T Y_{1.1}, q D T Y_{2.1}, q D T Y_{3.1}$ followed by the subjection of near isogenic lines in 60 multi-location trials across 18 and 6 locations in India and Nepal respectively with grain yield advantage under reproductive stage drought stress (Sandhu et al., 2019). QTL combinations in three introgression lines with $q D T Y_{2.2}, q D T Y_{4.1}, q D T Y_{10.1}$ and two introgression lines with $q D T Y_{2.2}$ and $q D T Y_{4.1}$ in IR64 background resulted in differential yield advantage during drought stress at the reproductive stage and the yield was similar to IR64 under non-stress conditions (Swamy et al., 2013). However, the effect size of the identified QTLs is very important for their exploitation in $\mathrm{MAB}$, as QTL with too large or too small effect size poses difficult handling in MAB (Cobb et al., 2019). Approaches such as bulk segregant analysis (BSA) and selective genotyping eliminates the selection of small effect size QTLs hence can be used as much effective, cost saving as well as a highly efficient genotyping approach (Vikram et al., 2011; Ghimire et al., 2012). Breeding-based approaches have then been successively exploited for drought tolerance in rice by taking grain yield as a criterion for selection (Bernier et al., 2007; Ghimire et al., 2012).

Drought acclimation in rice is highly dependent on root traits and the genetic variability in the physiology of root is linked with the plant productivity during drought stress (Dash et al., 2017). Introgressive hybridisation of DRO1, a rice QTL that controls root architecture during drought stress and improves drought avoidance, by continuous backcross with IR64 lines enhances drought resistance (Uga et al., 2013). Root traits-mediated drought tolerance has been observed in Kalinga III (an Indian upland rice variety) by introgression of 4 major QTLs for root traits such as length, thickness, penetration from Azucena (a tropical japonica rice variety) through markerassisted back-cross (MABC) breeding out of which QTL introgressed on chromosome 9 (QTL9) at target segment RM242-RM201 significantly enhances root length (Steele et al., 2006). Further, the field testing of near isogenic lines revealed $\mathrm{G} \times \mathrm{E}$ interaction and ineffectiveness of QTL9 on grain or straw yield, suggesting a possibility that introgressed genes might be involved in partitioning of biomass between root and shoot, while not in grains (Steele et al., 2007).

While physiological/secondary traits are less exploited due to difficulty in phenotyping and high $\mathrm{G} \times \mathrm{E}$ interaction and are mainly hindered by poor understanding of secondary traits. Hu et al. (2013) used the Oryza rufipogon genotype Dongxiang as a donor to develop backcross inbred lines (BILs) in the background of Oryza sativa as a recurrent parent and observed increased drought tolerance in introgressed lines with several physiological traits. Further, biological mechanism pertaining to drought resistance within rice was analysed by identification of conserved miRNAs underlying drought stress responses in wildtype rice cultivar Oryza rufipogon Griff., Dongxiang (Zhang et al., 2015).

\section{3 | CONCLUSION AND FUTURE PROSPECTS}

Drought stress causes various adversities in rice plants and affects their growth and yield. In rice plants, drought stress leads to detrimental 
effects on morphological and physiological traits. However, rice plants undergo various drought acclimation mechanisms to adapt to the adversities and enhance their growth and development. Alterations in root and shoot traits accompanied by mineral nutrient uptake and reduced water loss through transpiration are some of the morphophysiological adaptations undertaken by rice plants. Moreover, the involvement of plant AQPs in the modification of plant traits to facilitate the plasticity of rice plants during drought conditions enhances its value. Additionally, phytohormones also attenuates stress-induced adversities among which ABA is the central and the major mediator that counteracts the deleterious effects of drought stress in rice either independently or by crosstalk with other major phytohormones.

Since drought stress is the widespread constraint affecting agricultural sustainability thus the development of drought-tolerant rice varieties is needed to feed the growing population dependent on rice. Multi-dimensional approaches including genome editing, transgenic development and breeding-based approaches well supported by omics tools have been exploited to enhance rice drought tolerance. Both breeding-based approaches and/or transgenics have been continuously evolving with advancements in the identification of drought related trait loci and candidate genes respectively, with reinforcements of omics tools (genomics, proteomics and metabolomics). Moreover, genome editing using CRISPR/Cas system and TALENs have emerged as effective strategies to cope up with the effects of drought stress and improve the yield and nutritional status of rice. Several major drought responsive genes have been identified and characterised, however many are still under the hidden zone. Identification and functional characterisation of the unknown QTLs and the drought tolerance associated genes/proteins/metabolites may be utilised for developing more drought-tolerant rice cultivar.

\section{ACKNOWLEDGEMENTS}

The authors acknowledge all the contributors in the field of droughtrice and apologies if any work is/are not cited because of the space limitation. In addition, M. Iqbal R. Khan acknowledges the financial support by UGC-start-up grant (No. F. 30-482/2019 [BSR]). Sudhakar R. Palakolanu acknowledges the financial support by SERB under Extramural Research (EMR) Grant No. EMR/2016/006726.

\section{DATA AVAILABILITY STATEMENT}

Research data availability and/or sharing is not applicable to this review article.

\section{ORCID}

M. Iqbal R. Khan (D) https://orcid.org/0000-0002-7697-5723

\section{REFERENCES}

Abreu, F.R.M., Dedicova, B., Vianello, R.P., Lanna, A.C., de Oliveira, J.A.V., Vieira, A.F., et al. (2018) Overexpression of a phospholipase (OsPLD 1 ) for drought tolerance in upland rice (Oryza sativa L.). Protoplasma, 255, 1751-1761.

Agarwal, P., Arora, R., Ray, S., Singh, A.K., Singh, V.P., Takatsuji, H., et al. (2007) Genome-wide identification of $\mathrm{C}_{2} \mathrm{H}_{2}$ zinc-finger gene family in rice and their phylogeny and expression analysis. Plant Molecular Biology, 65, 467-485.

Ahmad, I., Mian, A. \& Maathuis, F.J. (2016) Overexpression of the rice AKT1 potassium channel affects potassium nutrition and rice drought tolerance. Journal of Experimental Botany, 67, 2689-2698.

Ahmar, S., Gill, R.A., Jung, K.-H., Faheem, A., Qasim, M.U., Mubeen, M., et al. (2020) Conventional and molecular techniques from simple breeding to speed breeding in crop plants: recent advances and future outlook. International Journal of Molecular Sciences, 21, 2590.

Aleman, F., Yazaki, J., Lee, M., Takahashi, Y., Kim, A.Y., Li, Z., et al. (2016) An ABA-increased interaction of the PYL6 ABA receptor with MYC2 transcription factor: A putative link of $A B A$ and JA signaling. Scientific Reports, 6, 28941.

Ansari, W.A., Chandanshive, S.U., Bhatt, V., Nadaf, A.B., Vats, S., Katara, J.L., et al. (2020) Genome editing in cereals: approaches, applications and challenges. International Journal of Molecular Sciences, 21, 4040.

Babu, R.C., Shashidhar, H.E., Lilley, J.M., Thanh, N.D., Ray, J.D., Sadasivam, S., et al. (2001) Variation in root penetration ability, osmotic adjustment and dehydration tolerance among accessions of rice adapted to rainfed lowland and upland ecosystems. Plant Breeding, 120, 233-238.

Babu, R.C., Zhang, J., Blum, A., Ho, T.H.D., Wu, R. \& Nguyen, H.T. (2004) HVA1, a LEA gene from barley confers dehydration tolerance in transgenic rice (Oryza sativa L.) via cell membrane protection. Plant Science, 166, 855-862.

Bae, H., Kim, S.K., Cho, S.K., Kang, B.G. \& Kim, W.T. (2011) Overexpression of OsRDCP1, a rice RING domain-containing E3 ubiquitin ligase, increased tolerance to drought stress in rice (Oryza sativa L.). Plant Science, 180, 775-782.

Bañuelos, M.A., Garciadeblas, B., Cubero, B. \& Rodrìguez-Navarro, A. (2002) Inventory and functional characterization of the HAK potassium transporters of rice. Plant Physiology, 130, 784-795.

Bardhan, K., Patel, D.S., Patel, D.P. (2018) Revealing the effects of potassium on rice roots under moisture stress. BC 102

Bernier, J., Kumar, A., Ramaiah, V., Spaner, D. \& Atlin, G. (2007) A largeeffect QTL for grain yield under reproductive-stage drought stress in upland rice. Crop Science, 47, 507-518.

Bista, D.R., Heckathorn, S.A., Jayawardena, D.M., Mishra, S. \& Boldt, J.K. (2018) Effects of drought on nutrient uptake and the levels of nutrient-uptake proteins in roots of drought-sensitive and -tolerant grasses. Plants (Basel), 7, 28.

Capell, T., Escobar, C., Liu, H., Burtin, D., Lepri, O. \& Christou, P. (1998) Over-expression of the oat arginine decarboxylase cDNA in transgenic rice (Oryza sativa L.) affects normal development patterns in vitro and results in putrescine accumulation in transgenic plants. Theoretical and Applied Genetics, 97, 246-254.

Chang, Z., Yan, W., Liu, D., Chen, Z., Xie, G., Lu, J., et al. (2015) Research progress on CRISPR/Cas. Journal of Agricultural Biotechnology, 23, 1196-1206.

Chen, G., Liu, C., Gao, Z., Zhang, Y., Jiang, H., Zhu, L., et al. (2017) OsHAK1, a high-affinity potassium transporter, positively regulates responses to drought stress in rice. Frontiers in Plant Science, 8, 1885.

Chen, J., Qi, T., Hu, Z., Fan, X., Zhu, L., Yin, X., et al. (2019) OsNAR2.1 positively regulates drought tolerance and grain yield under drought stress conditions in rice. Frontiers in Plant Science, 10, 197.

Chen, K., Li, G.J., Bressan, R.A., Song, C.P., Zhu, J.K. \& Zhao, Y. (2020) Abscisic acid dynamics, signaling, and functions in plants. Journal of Integrative Plant Biology, 62, 25-54.

Chen, W., Yao, X., Cai, K. \& Chen, J. (2011) Silicon alleviates drought stress of rice plants by improving plant water status, photosynthesis and mineral nutrient absorption. Biological Trace Element Research, 142, 67-76.

Choudhary, M.K., Basu, D., Datta, A., Chakraborty, N. \& Chakraborty, S. (2009) Dehydration-responsive nuclear proteome of rice (Oryza sativa 
L.) illustrates protein network, novel regulators of cellular adaptation, and evolutionary perspective. Molecular \& Cellular Proteomics, 8, 1579-1598.

Chung, P.J., Jung, H., Do Choi, Y. \& Kim, J.K. (2018) Genome-wide analyses of direct target genes of four rice NAC-domain transcription factors involved in drought tolerance. BMC Genomics, 19, 40.

Cobb, J.N., Biswas, P.S. \& Platten, J.D. (2019) Back to the future: revisiting MAS as a tool for modern plant breeding. Theoretical and Applied Genetics, 132, 647-667.

Cobb, J.N., DeClerck, G., Greenberg, A., Clark, R. \& McCouch, S. (2013) Next-generation phenotyping: requirements and strategies for enhancing our understanding of genotype-phenotype relationships and its relevance to crop improvement. Theoretical and Applied Genetics, 126, 867-887.

Comas, L.H., Becker, S.R., Cruz, V.M., Byrne, P.F. \& Dierig, D.A. (2013) Root traits contributing to plant productivity under drought. Frontiers in Plant Science, 4, 442.

Cui, L.H., Byun, M.Y., Oh, H.G., Kim, S.J., Lee, J., Park, H., et al. (2020) Poaceae type II galactinol synthase 2 from Antarctic flowering plant Deschampsia antarctica and rice improves cold and drought tolerance by accumulation of raffinose family oligosaccharides in transgenic rice plants. Plant \& Cell Physiology, 61, 88-104.

Cui, Y., Li, M., Yin, X., Song, S., Xu, G., Wang, M., et al. (2018) OsDSSR1, a novel small peptide, enhances drought tolerance in transgenic rice. Plant Science, 270, 85-96.

Cui, Y., Wang, M., Zhou, H., Li, M., Huang, L., Yin, X., et al. (2016) OsSGL, a novel DUF1645 domain-containing protein, confers enhanced drought tolerance in transgenic rice and Arabidopsis. Frontiers in Plant Science, 7, 2001.

Dash, G.K., Barik, M., Debata, A.K., Baig, M.J. \& Swain, P. (2017) Identification of most important rice root morphological markers in response to contrasting moisture regimes under vegetative stage drought. Acto Physiologiae Plantarum, 39, 8

Dash, P.K., Rai, R., Rai, V. \& Pasupalak, S. (2018) Drought induced signaling in rice: delineating canonical and non-canonical pathways. Frontiers in Chemistry, 6, 264

Daszkowska-Golec, A. \& Szarejko, I. (2013) Open or close the gatestomata action under the control of phytohormones in drought stress conditions. Frontiers in Plant Science, 4, 138.

de Ollas, C. \& Dodd, I.C. (2016) Physiological impacts of ABA-JA interactions under water-limitation. Plant Molecular Biology, 91 641-650.

Degenkolbe, T., Do, P.T., Kopka, J., Zuther, E., Hincha, D.K. \& Köhl, K.I. (2013) Identification of drought tolerance markers in a diverse population of rice cultivars by expression and metabolite profiling. PLOS ONE, 8, e63637.

Devi, S.H. \& Kar, M. (2013) Amelioration of moisture stress effect by $\mathrm{CaCl}_{2}$ pre-treatment in upland rice. Indian Journal of Plant Physiology, 18, 384-387.

Dhakarey, R., Raorane, M.L., Treumann, A., Peethambaran, P.K., Schendel, R.R., Sahi, V.P., et al. (2017) Physiological and proteomic analysis of the rice mutant $\mathrm{cpm} 2$ suggests a negative regulatory role of jasmonic acid in drought tolerance. Frontiers in Plant Science, 8 , 1903.

Ding, L., Gao, C., Li, Y., Li, Y., Zhu, Y., Xu, G., et al. (2015) The enhanced drought tolerance of rice plants under ammonium is related to aquaporin (AQP). Plant Science, 234, 14-21.

Ding, L., Uehlein, N., Kaldenhoff, R., Guo, S., Zhu, Y. \& Kai, L. (2019) Aquaporin PIP2; 1 affects water transport and root growth in rice (Oryza sativa L.). Plant Physiology and Biochemistry, 139, 152-160.

Dittrich, M., Mueller, H.M., Bauer, H., Peirats-Llobet, M., Rodriguez, P.L., Geilfus, C.M., et al. (2019) The role of Arabidopsis ABA receptors from the PYR/PYL/RCAR family in stomatal acclimation and closure signal integration. Nature Plants, 5, 1002-1011.
Du, H., Wang, N., Cui, F., Li, X., Xiao, J. \& Xiong, L. (2010) Characterization of a $\beta$-carotene hydroxylase gene DSM2 conferring drought and oxidative stress resistance by increasing xanthophylls and $A B A$ synthesis in rice. Plant Physiology, 154, 1304-1318.

Du, H., Wu, N., Chang, Y., Li, X., Xiao, J. \& Xiong, L. (2013) Carotenoid deficiency impairs $A B A$ and IAA biosynthesis and differentially affects drought and cold tolerance in rice. Plant Molecular Biology, 83, 475-488.

Du, H., Wu, N., Cui, F., You, L., Li, X. \& Xiong, L. (2014) A homolog of ethylene overproducer, OsETOL1, differentially modulates drought and submergence tolerance in rice. The Plant Journal, 78, 834-849.

Du, H., Wu, N., Fu, J., Wang, S., Li, X., Xiao, J., et al. (2012) A GH3 family member, OsGH3-2, modulates auxin and abscisic acid levels and differentially affects drought and cold tolerance in rice. Journal of Experimental Botany, 63, 6467-6480.

Duan, J. \& Cai, W. (2012) OsLEA3-2, an abiotic stress induced gene of rice plays a key role in salt and drought tolerance. PLoS ONE, 7, e45117.

Dusenge, M.E., Duarte, A.G. \& Way, D.A. (2019) Plant carbon metabolism and climate change: elevated $\mathrm{CO}_{2}$ and temperature impacts on photosynthesis, photorespiration and respiration. The New Phytologist, 221, 32-49.

Elbasan, F., Ozfidan-Konakci, C., Yildiztugay, E. \& Kucukoduk, M. (2020) Rare-earth element scandium improves stomatal regulation and enhances salt and drought stress tolerance by up-regulating antioxidant responses of Oryza sativa. Plant Physiology and Biochemistry, 152, 157-169.

Emam, M.M., Khattab, H., Helal, N.M. \& Deraz, A.E. (2014) Effect of selenium and silicon on yield quality of rice plant grown under drought stress. Australian Journal of Crop Science, 8, 596-605.

Fang, Y., Liao, K., Du, H., Xu, Y., Song, H., Li, X., et al. (2015) A stressresponsive NAC transcription factor SNAC3 confers heat and drought tolerance through modulation of reactive oxygen species in rice. Journal of Experimental Botany, 66, 6803-6817.

Farooq, M., Kobayashi, N., Ito, O., Wahid, A. \& Serraj, R. (2010) Broader leaves result in better performance of indica rice under drought stress. Journal of Plant Physiology, 167, 1066-1075.

Feller, U. (2016) Drought stress and carbon assimilation in a warming climate: reversible and irreversible impacts. Journal of Plant Physiology, 203, 84-94.

Food and Agriculture Organization of the United Nations (FAO). (2016) Rice market monitor. 19. Available at: http://www.fao.org/fileadmin/ templates/est/COMM_MARKETS_MONITORING/Rice/Images/RMM/ RMM_APR16.pdf (accessed April 2016).

Food and Agriculture Organization of the United Nations (FAO). (2018) The impact of disasters and crises on agriculture and food security. Available at: http://www.fao.org/3//8656EN/i8656en.pdf (accessed March 2018).

French, P., Matsuyuki, H. \& Ueno, H. (1990) Paclobutrazol: control of lodging in Japanese paddy rice. In: Grayson, B.T., Green, M.B. \& Copping, L.G. (Eds.) Proceedings of the conference held by the society of chemical industry: pest management in rice. London: Elsevier Applied Science Publishers Ltd., pp. 474-485.

Fu, J., Wu, H., Ma, S., Xiang, D., Liu, R. \& Xiong, L. (2017) OsJAZ1 attenuates drought resistance by regulating $\mathrm{JA}$ and $\mathrm{ABA}$ signaling in Rice. Frontiers in Plant Science, 8, 2108.

Fukai, S. \& Cooper, M. (1995) Development of drought resistant cultivars using physio-morphological traits in rice. Field Crops Research, 40, 67-86.

Ganguly, M., Roychoudhury, A., Sengupta, D.N., Datta, S.K. \& Datta, K. (2020) Independent overexpression of OsRab16A and AtDREB1A exhibit enhanced drought tolerance in transgenic aromatic rice variety Pusa Sugandhi 2. Journal of Plant Biochemistry and Biotechnology, 29, 503-517.

Gao, S., Fang, J., Xu, F., Wang, W., Sun, X., Chu, J., et al. (2014) CYTOKININ OXIDASE/DEHYDROGENASE4 integrates cytokinin and auxin 
signaling to control rice crown root formation. Plant Physiology, 165, 1035-1046.

Gao, T., Wu, Y., Zhang, Y., Liu, L., Ning, Y., Wang, D., et al. (2011) OsSDIR1 overexpression greatly improves drought tolerance in transgenic rice. Plant Molecular Biology, 76, 145-156.

Gao, W., Long, L., Tian, X., Xu, F., Liu, J., Singh, P.K., et al. (2017) Genome editing in cotton with the CRISPR/Cas9 system. Frontiers in Plant Science, 8, 1364.

Gasparis, S., Kała, M., Przyborowski, M., Łyżnik, L.A., Orczyk, W. \& Nadolska-Orczyk, A. (2018) A simple and efficient CRISPR/Cas9 platform for induction of single and multiple, heritable mutations in barley (Hordeum vulgare L.). Plant Methods, 14, 111.

Gaudin, A.C., Henry, A., Sparks, A.H. \& Slamet-Loedin, I.H. (2013) Taking transgenic rice drought screening to the field. Journal of Experimental Botany, 64, 109-117.

Gayen, D., Barua, P., Lande, N.V., Varshney, S., Sengupta, S., Chakraborty, S., et al. (2019) Dehydration-responsive alterations in the chloroplast proteome and cell metabolomic profile of rice reveals key stress adaptation responses. Environmental and Experimental Botany, $160,12-24$.

Ghimire, K.H., Lenie, A.Q., Vikram, P., Swamy, B.P.M., Dixit, S., Ahmed, H., et al. (2012) Identification and mapping of a QTL $\left(q D T Y_{1.1}\right)$ with a consistent effect on grain yield under drought. Field Crops Research, 131, 88-96.

Ghosh, A., Shah, M.N.A., Jui, Z.S., Saha, S., Fariha, K.A. \& Islam, T. (2018) Evolutionary variation and expression profiling of Isopentenyl transferase gene family in Arabidopsis thaliana L. and Oryza sativa L. Plant Gene, 15, 15-27.

Goff, S.A., Ricke, D., Lan, T.H., Presting, G., Wang, R., Dunn, M., et al. (2002) A draft sequence of the rice genome (Oryza sativa L. ssp. japonica). Science, 296, 92-100.

Gonzalez, R.M. \& lusem, N.D. (2014) Twenty years of research on Asr (ABA-stress-ripening) genes and proteins. Planta, 239, 941-949.

Gosti, F., Beaudoin, N., Serizet, C., Webb, A.A., Vartanian, N. \& Giraudat, J. (1999) $A B I 1$ protein phosphatase $2 C$ is a negative regulator of abscisic acid signaling. Plant Cell, 11, 1897-1909.

Gowda, V.R.P., Henry, A., Vadez, V., Shashidhar, H.E. \& Serraj, R. (2012) Water uptake dynamics under progressive drought stress in diverse accessions of the OryzaSNP panel of rice (Oryza sativa). Functional Plant Biology, 39, 402-411.

Gowda, V.R.P., Henry, A., Yamauchi, A., Shashidhar, H.E. \& Serraj, R. (2011) Root biology and genetic improvement for drought avoidance in rice. Field Crops Research, 122, 1-13.

Grondin, A., Mauleon, R., Vadez, V. \& Henry, A. (2015) Root aquaporins contribute to whole plant water fluxes under drought stress in rice (Oryza sativa L.). Plant, Cell \& Environment, 39, 347-365.

Guo, L., Wang, Z.Y., Lin, H., Cui, W.E., Chen, J., Liu, M., et al. (2006) Expression and functional analysis of the rice plasma-membrane intrinsic protein gene family. Cell Research, 16, 277-286.

Guo, S., Chen, G., Zhou, Y. \& Shen, Q. (2007) Ammonium nutrition increases photosynthesis rate under water stress at early development stage of rice (Oryza sativa L.). Plant and Soil, 296, 115-124.

Gupta, R., Min, C.W., Kim, S.W., Wang, Y., Agrawal, G.K., Rakwal, R., et al. (2015a) Comparative investigation of seed coats of brown- versus yellow-colored soybean seeds using an integrated proteomics and metabolomics approach. Proteomics, 15, 1706-1716.

Gupta, R., Min, C.W., Kim, Y.J. \& Kim, S.T. (2019) Identification of Msp1-induced signaling components in rice leaves by integrated proteomic and phosphoproteomic analysis. International Journal of Molecular Sciences, 20, 4135.

Gupta, R., Min, C.W., Kramer, K., Agrawal, G.K., Rakwal, R., Park, K.H., et al. (2018) A multi-omics analysis of Glycine max leaves reveals alteration in flavonoid and isoflavonoid metabolism upon ethylene and abscisic acid treatment. Proteomics, 18, e1700366.
Gupta, R., Wang, Y., Agrawal, G.K., Rakwal, R., Jo, I.H., Bang, K.H., et al. (2015b) Time to dig deep into the plant proteome: a hunt for lowabundance proteins. Frontiers in Plant Science, 6, 1-3.

Haider, I., Andreo-Jimenez, B., Bruno, M., Bimbo, A., Floková, K., Abuauf, H., et al. (2018) The interaction of strigolactones with abscisic acid during the drought response in rice. Journal of Experimental Botany, 69, 2403-2414.

Hamzelou, S., Pascovici, D., Kamath, K.S., Amirkhani, A., McKay, M., Mirzaei, M., et al. (2020) Proteomic responses to drought vary widely among eight diverse genotypes of rice (Oryza sativa). International Journal of Molecular Sciences, 21, 363.

Hanin, M., Brini, F., Ebel, C., Toda, Y., Takeda, S. \& Masmoudi, K. (2011) Plant dehydrins and stress tolerance: versatile proteins for complex mechanisms. Plant Signaling \& Behavior, 6, 1503-1509.

Hao, W. \& Lin, H.X. (2010) Toward understanding genetic mechanisms of complex traits in rice. Journal of Genetics and Genomics, 37, 653-666.

Harrison, M.A. (2012) Cross-talk between phytohormone signaling pathways under both optimal and stressful environmental conditions. In: Khan, $\mathrm{N}$. A., Nazar, R., Iqbal, N. \& Anjum, N.A. (Eds.) Phytohormones and Abiotic Stress Tolerance in Plants. Berlin, Heidelberg: Springer-Verlag, pp. 49-76.

Hasanuzzaman, M., Bhuyan, M.H.M.B., Nahar, K., Hossain, M.S., Mahmud, J.A., Hossen, M.S., et al. (2018) Potassium: a vital regulator of plant responses and tolerance to abiotic stresses. Agronomy, 8, 31.

Hazen, S.P., Pathan, M.S., Sanchez, A., Baxter, I., Dunn, M., Estes, B., et al. (2005) Expression profiling of rice segregating for drought tolerance QTLs using a rice genome array. Functional \& Integrative Genomics, 5, 104-116.

He, Y., Hao, Q., Li, W., Yan, C., Yan, N. \& Yin, P. (2014) Identification and characterization of ABA receptors in Oryza sativa. PLoS ONE, 9, e95246.

Hedden, P. (2003) The genes of the green revolution. Trends in Genetics, 19, 5-9.

Henry, A., Cal, A.J., Batoto, T.C., Torres, R.O. \& Serraj, R. (2012) Root attributes affecting water uptake of rice (Oryza sativa) under drought. Journal of Experimental Botany, 63, 4751-4763.

Henry, A., Dixit, S., Mandal, N.P., Anantha, M.S., Torres, R. \& Kumar, A. (2014) Grain yield and physiological traits of rice lines with the drought yield QTL $9 D T Y_{12.1}$ showed different responses to drought and soil characteristics in upland environments. Functional Plant Biology, 41, 1066-1077.

Henry, A., Gowda, V.R., Torres, R.O., McNally, K.L. \& Serraj, R. (2011) Variation in root system architecture and drought response in rice (Oryza sativa): phenotyping of the OryzaSNP panel in rainfed lowland fields. Field Crops Research, 120, 205-214.

Henry, A., Swamy, B.P.M., Dixit, S., Torres, R.D., Batoto, T.C., Manalili, M., et al. (2015) Physiological mechanisms contributing to the QTLcombination effects on improved performance of IR64 rice NILs under drought. Journal of Experimental Botany, 66, 1787-1799.

Hong, Y., Zhang, H., Huang, L., Li, D. \& Song, F. (2016) Overexpression of a stress-responsive NAC transcription factor gene ONAC022 improves drought and salt tolerance in rice. Frontiers in Plant Science, 7, 4.

Hu, B.L., Yang, P., Wan, Y., Li, X., Luo, S.Y., Luo, X.D., et al. (2013) Comprehensive assessment of drought resistance of BILs population derived from Dongxiang wild rice (Oryza rufipogon Griff.) at seedling stage and its genetic analysis. Plant Genetic Resources, 14, 249-256.

Hu, H., Dai, M., Yao, J., Xiao, B., Li, X., Zhang, Q., et al. (2006) Overexpressing a NAM, ATAF, and CUC (NAC) transcription factor enhances drought resistance and salt tolerance in rice. Proceedings of the National Academy of Sciences, 103, 12987-12992.

Hu, H. \& Xiong, L. (2014) Genetic engineering and breeding of droughtresistant crops. Annual Review of Plant Biology, 65, 715-741.

Hu, Y., Wu, Q., Peng, Z., Sprague, S.A., Wang, W., Park, J., et al. (2017) Silencing of OsGRXS17 in rice improves drought stress tolerance by modulating ROS accumulation and stomatal closure. Scientific Reports, 7,15950 
Huang, D., Wu, W., Abrams, S.R. \& Cutler, A.J. (2008) The relationship of drought-related gene expression in Arabidopsis thaliana to hormonal and environmental factors. Journal of Experimental Botany, 59, 2991-3007.

Huang, L., Hong, Y., Zhang, H., Li, D. \& Song, F. (2016) Rice NAC transcription factor ONAC095 plays opposite roles in drought and cold stress tolerance. BMC Plant Biology, 16, 203.

Huang, X., Hou, L., Meng, J., You, H., Li, Z., Gong, Z., et al. (2018) The antagonistic action of abscisic acid and cytokinin signaling mediates drought stress response in Arabidopsis. Molecular Plant, 11, 970-982.

Ingram, K.T., Bueno, F.D., Namuco, O.S., Yambao, E.B., Beyrouty, C.A (1994) Rice root traits for drought resistance and their genetic variation.

Iseki, K., Homma, K., Shiraiwa, T., Jongdee, B. \& Mekwatanakarn, P. (2014) The effects of cross-tolerance to oxidative stress and drought stress on rice dry matter production under aerobic conditions. Field Crops Research, 163, 18-23.

Islam, M.Z., Khalequzzaman, M., Prince, M.F.R.K., Siddique, M.A., Rashid, E. S.M.H., Ahmed, M.S.U., et al. (2018) Diversity and population structure of red rice germplasm in Bangladesh. PLoS ONE, 13, e0196096.

Ito, Y., Katsura, K., Maruyama, K., Taji, T., Kobayashi, M., Seki, M., et al. (2006) Functional analysis of rice DREB1/CBF-type transcription fac tors involved in cold-responsive gene expression in transgenic rice. Plant \& Cell Physiology, 47, 141-153.

Jaiswal, D.K., Ray, D., Choudhary, M.K., Subba, P., Kumar, A., Verma, J., et al. (2013) Comparative proteomics of dehydration response in the rice nucleus: new insights into the molecular basis of genotypespecific adaptation. Proteomics, 13, 3478-3497.

Jeong, J.S., Kim, Y.S., Baek, K.H., Jung, H., Ha, S.H., Do Choi, Y., et al. (2010) Root-specific expression of OsNAC10 improves drought tolerance and grain yield in rice under field drought conditions. Plant Physiology, 153, 185-197.

Jeong, J.S., Kim, Y.S., Redillas, M.C., Jang, G., Jung, H., Bang, S.W., et al. (2013) OsNAC5 overexpression enlarges root diameter in rice plants leading to enhanced drought tolerance and increased grain yield in the field. Plant Biotechnology Journal, 11, 101-114.

Jin, Y., Pan, W., Zheng, X., Cheng, X., Liu, M., Ma, H., et al. (2018) OsERF101, an ERF family transcription factor, regulates drought stress response in reproductive tissues. Plant Molecular Biology, 98, 51-65.

Joo, J., Choi, D.H., Lee, Y.H., Seo, H.S. \& Song, S.I. (2019a) The rice SUMO conjugating enzymes OsSCE1 and OsSCE3 have opposing effects on drought stress. Journal of Plant Physiology, 240, 152993.

Joo, J., Lee, Y.H. \& Song, S.I. (2019b) OsbZIP42 is a positive regulator of ABA signaling and confers drought tolerance to rice. Planta, 249, 1521-1533.

Jung, H., Chung, P.J., Park, S.H., Redillas, M.C.F.R., Kim, Y.S., Suh, J.W., et al. (2017) Overexpression of OsERF48 causes regulation of OsCML16, a calmodulin-like protein gene that enhances root growth and drought tolerance. Plant Biotechnology Journal, 15, 1295-1308.

Jung, H., Lee, D.K., Choi, Y.D. \& Kim, J.K. (2015) OsIAA6, a member of the rice Aux/IAA gene family, is involved in drought tolerance and tiller outgrowth. Plant Science, 236, 304-312.

Kaashyap, M., Ford, R., Kudapa, H., Jain, M., Edwards, D., Varshney, R., et al. (2018) Differential regulation of genes involved in root morphogenesis and cell wall modification is associated with salinity tolerance in chickpea. Scientific Reports, 8, 4855.

Kano-Nakata, M., Gowda, V.R., Henry, A., Serraj, R., Inukai, Y., Fujita, D. et al. (2013) Functional roles of the plasticity of root system development in biomass production and water uptake under rainfed lowland conditions. Field Crops Research, 144, 288-296.

Kazan, K. (2015) Diverse roles of jasmonates and ethylene in abiotic stress tolerance. Trends in Plant Science, 20, 219-229.

Ke, Y., Han, G., He, H. \& Li, J. (2009) Differential regulation of proteins and phosphoproteins in rice under drought stress. Biochemical and Biophysical Research Communications, 379, 133-138.
Kim, E.H., Park, S.H. \& Kim, J.K. (2009) Methyl jasmonate triggers loss of grain yield under drought stress. Plant Signaling \& Behavior, 4 348-349.

Kim, S.T., Kim, S.G., Agrawal, G.K., Kikuchi, S. \& Rakwal, R. (2014) Rice proteomics: a model system for crop improvement and food security. Proteomics, 14, 593-610.

Kim, T.H., Böhmer, M., Hu, H., Nishimura, N. \& Schroeder, J.I. (2010) Guard cell signal transduction network: advances in understanding abscisic acid, $\mathrm{CO}_{2}$ and $\mathrm{Ca}^{2+}$ signaling. Annual Review of Plant Biology, 61, 561-591.

Koevoets, I.T., Venema, J.H., Elzenga, J.T. \& Testerink, C. (2016) Roots withstanding their environment: exploiting root system architecture responses to abiotic stress to improve crop tolerance. Frontiers in Plant Science, 7, 1335.

Komor, A.C., Kim, Y.B., Packer, M.S., Zuris, J.A. \& Liu, D.R. (2016) Programmable editing of a target base in genomic DNA without double stranded DNA cleavage. Nature, 533, 420-424.

Kumar, S., Dwivedi, S.K., Basu, S., Kumar, G., Mishra, J.S., Koley, T.K., et al. (2020) Anatomical, agro-morphological and physiological changes in rice under cumulative and stage specific drought conditions prevailed in eastern region of India. Field Crops Research, 245, 107658.

Kuromori, T., Seo, M. \& Shinozaki, K. (2018) ABA transport and plant water stress responses. Trends in Plant Science, 23, 513-522.

Lawlor, D.W. (2002) Limitation to photosynthesis in water-stressed leaves: stomata vs. metabolism and the role of ATP. Annals of Botany, 89, 871-885.

Laxalt, A.M., García-Mata, C. \& Lamattina, L. (2016) The dual role of nitric oxide in guard cells: promoting and attenuating the ABA and phospholipid derived signals leading to the stomatal closure. Frontiers in Plant Science, 7, 476.

Lee, B.R., Jung, W.J., Lee, B.H., Avice, J.C., Ourry, A. \& Kim, T.H. (2008) Kinetics of drought-induced pathogenesis-related proteins and its physiological significance in white clover leaves. Physiologia Plantarum, 132, 329-337.

Lee, D.K., Jung, H., Jang, G., Jeong, J.S., Kim, Y.S., Ha, S.H., et al. (2016) Overexpression of the OsERF71 transcription factor alters rice root structure and drought resistance. Plant Physiology, 172, 575-588.

Lee, D.K., Shim, G.P.J., Jeong, J.S., Jang, G., Bang, S.W., Jung, H., et al. (2017) The rice OsNAC6 transcription factor orchestrates multiple molecular mechanisms involving root structural adaptions and nicotianamine biosynthesis for drought tolerance. Plant Biotechnology Journal, 15, 754-764.

Li, A., Jia, S., Yobi, A., Ge, Z., Sato, S., Zhang, C., et al. (2018a) Editing of an alpha-kafirin gene family increases digestibility and protein quality in sorghum. Plant Physiology, 177, 1425-1438.

Li, H.W., Zang, B.S., Deng, X.W. \& Wang, X.P. (2011) Overexpression of the trehalose-6-phosphate synthase gene OsTPS1 enhances abiotic stress tolerance in rice. Planta, 234, 1007-1018.

Li, J., Guo, X., Zhang, M., Wang, X., Zhao, Y., Yin, Z., et al. (2018b) OsERF71 confers drought tolerance via modulating $A B A$ signaling and proline biosynthesis. Plant Science, 270, 131-139.

Li, J., Li, Y., Yin, Z., Jiang, J., Zhang, M., Guo, X., et al. (2017) OsASR5 enhances drought tolerance through a stomatal closure pathway associated with $\mathrm{ABA}$ and $\mathrm{H}_{2} \mathrm{O}_{2}$ signalling in rice. Plant Biotechnology Journal, 15, 183-196.

Li, R., Wang, J., Li, S., Zhang, L., Qi, C., Weeda, S., et al. (2016) Plasma membrane intrinsic proteins SIPIP2;1, SIPIP2;7 and SIPIP2;5 conferring enhanced drought stress tolerance in tomato. Scientific Reports, 6, 31814

Li, X., Chang, Y., Ma, S., Shen, J., Hu, H. \& Xiong, L. (2019b) Genome-wide identification of SNAC1-targeted genes involved in drought response in rice. Frontiers in Plant Science, 10, 982.

Li, X., Lawas, L.M.F., Malo, R., Glaubitz, U., Erban, A., Mauleon, R., et al. (2015) Metabolic and transcriptomic signatures of rice floral organs reveal sugar starvation as a factor in reproductive failure under heat and drought stress. Plant, Cell \& Environment, 38, 2171-2192. 
Li, X., Zhang, Q., Yang, X., Han, J. \& Zhu, Z. (2019a) OsANN3, a calciumdependent lipid binding annexin is a positive regulator of $A B A-$ dependent stress tolerance in rice. Plant Science, 284, 212-220.

Lian, H.L., Yu, X., Ye, Q., Ding, X.S., Kitagawa, Y., Kwak, S.S., et al. (2004) The role of aquaporin RWC3 in drought avoidance in rice. Plant \& Cell Physiology, 45, 481-489.

Liu, C., Mao, B., Ou, S., Wang, W., Liu, L., Wu, Y., et al. (2014) OsbZIP71, a bZIP transcription factor, confers salinity and drought tolerance in rice. Plant Molecular Biology, 84, 19-36.

Liu, J., Sun, X., Liao, W., Zhang, J., Liang, J. \& Xu, W. (2019) Involvement of OsGF14b adaptation in the drought resistance of rice plants. Rice, 12, 82.

Liu, J.-X. \& Bennett, J. (2011) Reversible and irreversible drought-induced changes in the anther proteome of rice (Oryza sativa L.) genotypes IR64 and Moroberekan. Molecular Plant, 4, 59-69.

Lou, D., Wang, H., Liang, G. \& Yu, D. (2017) OsSAPK2 confers abscisic acid sensitivity and tolerance to drought stress in rice. Frontiers in Plant Science, 8, 993.

Lu, G., Gao, C., Zheng, X. \& Han, B. (2009) Identification of OsbZIP72 as a positive regulator of $A B A$ response and drought tolerance in rice. Planta, 229, 605-615.

Ma, X., Xia, H., Liu, Y., Wei, H., Zheng, X., Song, C., et al. (2016) Transcriptomic and metabolomic studies disclose key metabolism pathways contributing to well-maintained photosynthesis under the drought and the consequent drought-tolerance in rice. Frontiers in Plant Science, 7, 1886.

Ma, Y., Szostkiewicz, I., Korte, A., Moes, D., Yang, Y., Christmann, A., et al. (2009) Regulators of PP2C phosphatase activity function as abscisic acid sensors. Science, 324, 1064-1068.

Markandeya, G., Babu, P.R., Reddy Lachagari, V.B., Alex Feltus, F., Paterson, A.H. \& Reddy Arjula, R. (2005) Functional genomics of drought stress response in rice: transcript mapping of annotated unigenes of an indica rice (Oryza sativa L. cv. Nagina 22). Current Science, 89, 496-514.

Maurel, C., Simonneau, T. \& Sutka, M. (2010) The significance of roots as hydraulic rheostats. Journal of Experimental Botany, 61, 3191-3198.

Melandri, G., Prashar, A., Mccouch, S.R., Van Der Linden, G., Jones, H.G., Kadam, N., et al. (2020) Association mapping and genetic dissection of drought-induced canopy temperature differences in rice. Journal of Experimental Botany, 71, 1614-1627.

Meng, Q., Gupta, R., Min, C.W., Kim, J., Kramer, K., Wang, Y., et al. (2018) Label-free quantitative proteome data associated with MSP1 and flg22 induced signaling in rice leaves. Data in Brief, 20, 204-209.

Meng, Q., Gupta, R., Min, C.W., Kim, J., Kramer, K., Wang, Y., et al. (2019) A proteomic insight into the MSP1 and flg22 induced signaling in Oryza sativa leaves. Journal of Proteomics, 196, 120-130.

Mickelbart, M.V., Hasegawa, P.M. \& Bailey-Serres, J. (2015) Genetic mechanisms of abiotic stress tolerance that translate to crop yield stability. Nature Reviews. Genetics, 16, 237-251.

Ming, D.F., Pei, Z.F., Naeem, M.S., Gong, H.J. \& Zhou, W.J. (2012) Silicon alleviates PEG-induced water-deficit stress in upland rice seedlings by enhancing osmotic adjustment. Journal of Agronomy and Crop Science, 198, 14-26.

Minh-Thu, P.T., Kim, J.S., Chae, S., Jun, K.M., Lee, G.S., Kim, D.E., et al. (2018) A WUSCHEL homeobox transcription factor, OsWOX13, enhances drought tolerance and triggers early flowering in rice. Molecules and Cells, 41, 781.

Mirzaei, M., Pascovici, D., Atwell, B.J. \& Haynes, P.A. (2012a) Differential regulation of aquaporins, small GTPases and V-ATPases proteins in rice leaves subjected to drought stress and recovery. Proteomics, 12, 864-877.

Mirzaei, M., Soltani, N., Sarhadi, E., Pascovici, D., Keighley, T., Salekdeh, G. H., et al. (2012b) Shotgun proteomic analysis of long-distance drought signaling in rice roots. Journal of Proteome Research, 11, 348-358.

Mishra, R. \& Zhao, K. (2018) Genome editing technologies and their applications in crop improvement. Plant Biotechnology Report, 12, 57-68.
Mohd Zain, N.A. \& Ismail, M.R. (2016) Effects of potassium rates and types on growth, leaf gas exchange and biochemical changes in rice (Oryza sativa) planted under cyclic water stress. Agricultural Water Management, 164, 83-90.

Mumtaz, M.Z., Saqib, M., Abbas, G., Akhtar, J. \& Ul-Qamar, Z. (2020) Drought stress impairs grain yield and quality of rice genotypes by impaired photosynthetic attributes and K nutrition. Rice Science, 27, 5-9.

Muthurajan, R., Shobbar, Z.-S., Jagadish, S.V.K., Bruskiewich, R., Ismail, A., Leung, H., et al. (2011) Physiological and proteomic responses of rice peduncles to drought stress. Molecular Biotechnology, 48, 173-182.

Nada, R.M. \& Abogadallah, G.M. (2020) Contrasting root traits and native regulation of aquaporin differentially determine the outcome of overexpressing a single aquaporin (OsPIP2; 4) in two rice cultivars. Protoplasma, 257, 583-595.

Nahar, S., Sahoo, L. \& Tanti, B. (2018) Screening of drought tolerant rice through morpho-physiological and biochemical approaches. Biocatalysis and Agricultural Biotechnology, 15, 150-159.

Nakashima, K., Tran, L.S., Van Nguyen, D., Fujita, M., Maruyama, K., Todaka, D., et al. (2007) Functional analysis of a NAC-type transcription factor OsNAC6 involved in abiotic and biotic stress-responsive gene expression in rice. The Plant Journal, 51, 617-630.

Nakashima, K. \& Yamaguchi-Shinozaki, K. (2006) Regulons involved in osmotic stress-responsive and cold stress-responsive gene expression in plants. Physiologia Plantarum, 126, 62-71.

Nguyen, H.T., Babu, R.C. \& Blum, A. (1997) Breeding for drought resistance in rice: physiology and molecular genetics considerations. Crop Science, 37, 1426-1434.

Niones, J.M., Suralta, R.R., Inukai, Y. \& Yamauchi, A. (2012) Field evaluation on functional roles of root plastic responses on dry matter production and grain yield of rice under cycles of transient soil moisture stresses using chromosome segment substitution lines. Plant and Soil, 359, 107-120.

Nishiyama, R., Watanabe, Y., Fujita, Y., Le, D.T., Kojima, M., Werner, T., et al. (2011) Analysis of cytokinin mutants and regulation of cytokinin metabolic genes reveals important regulatory roles of cytokinins in drought, salt and abscisic acid responses, and abscisic acid biosynthesis. Plant Cell, 23, 2169-2183.

Pan, J., Li, Z., Wang, Q., Yang, L., Yao, F. \& Liu, W. (2020) An S-domain receptor-like kinase, OsESG1, regulates early crown root development and drought resistance in rice. Plant Science, 290, 110318.

Pandey, A., Rajamani, U., Verma, J., Subba, P., Chakraborty, N., Datta, A., et al. (2010) Identification of extracellular matrix proteins of rice (Oryza sativa L.) involved in dehydration-responsive network: a proteomic approach. Journal of Proteome Research, 9, 3443-3464.

Pandey, V. \& Shukla, A. (2015) Acclimation and tolerance strategies of rice under drought stress. Rice Science, 22, 147-161.

Pandit, E., Panda, R.K., Sahoo, A., Pani, D.R. \& Pradhan, S.K. (2020) Genetic relationship and structure analysis of root growth angle for improvement of drought avoidance in early and mid-early maturing rice genotypes. Rice Science, 27, 124-132.

Park, S.I., Kim, J.J., Shin, S.Y., Kim, Y.S. \& Yoon, H.S. (2020) ASR enhances environmental stress tolerance and improves grain yield by modulating stomatal closure in rice. Frontiers in Plant Science, 10, 1752.

Park, S.Y., Fung, P., Nishimura, N., Jensen, D.R., Fujii, H., Zhao, Y., et al. (2009) Abscisic acid inhibits type $2 C$ protein phosphatases via the PYR/PYL family of START proteins. Science, 324, 1068-1071.

Patra, B.C., Ray, S., Ngangkham, U. \& Mohapatra, T. (2016) Genetic and genomic resources for grain cereals improvement. Rice, 1-80.

Pattanagul, W. (2011) Exogenous abscisic acid enhances sugar accumulation in rice. Asian Journal of Plant Sciences, 10, 212-219.

Pawłowicz, I. \& Masajada, K. (2018) Aquaporins as a link between water relations and photosynthetic pathway in abiotic stress tolerance in plants. Gene, 687, 166-172.

Peleg, Z., Reguera, M., Tumimbang, E., Walia, H. \& Blumwald, E. (2011) Cytokinin-mediated source/sink modifications improve drought 
tolerance and increase grain yield in rice under water-stress. Plant Biotechnology Journal, 9, 747-758.

Plaza-Wüthrich, S., Blösch, R., Rindisbacher, A., Cannarozzi, G. \& Tadele, Z. (2016) Gibberellin deficiency confers both lodging and drought tolerance in small cereals. Frontiers in Plant Science, 7, 643.

Pornsiriwong, W., Estavillo, G.M., Chan, K.X., Tee, E.E., Dm, G., Crisp, P.A., et al. (2017) A chloroplast retrograde signal, 3'-phosphoadenosine $5^{\prime}$-phosphate, acts as a secondary messenger in abscisic acid signaling in stomatal closure and germination. elife, 6, e23361.

Qin, Q., Wang, Y., Huang, L., Du, F., Zhao, X., Li, Z., et al. (2020) A U-box E3 ubiquitin ligase OsPUB67 is positively involved in drought tolerance in rice. Plant Molecular Biology, 102, 89-107.

Rabello, F.R., Villeth, G.R.C., Rabello, A.R., Rangel, P.H., Guimarães, C.M., Huergo, L.F., et al. (2014) Proteomic analysis of upland rice (Oryza sativa L.) exposed to intermittent water deficit. The Protein Journal, 33 221-230.

Raman, A., Verulkar, S.B., Mandal, N.P., Variar, M., Shukla, V.D., Dwivedi, J. L., et al. (2012) Drought yield index to select high yielding rice lines under different drought stress severities. Rice, 5, 1-12.

Ranathunge, K., Lin, J., Steudle, E. \& Schreiber, L. (2011) Stagnant deoxygenated growth enhances root suberization and lignifications, but differentially affects water and $\mathrm{NaCl}$ permeabilities in rice (Oryza sativa L.) roots. Plant, Cell \& Environment, 34, 1223-1240.

Redillas, M.C., Jeong, J.S., Kim, Y.S., Jung, H., Bang, S.W., Choi, Y.D., et al. (2012) The overexpression of OsNAC9 alters the root architecture of rice plants enhancing drought resistance and grain yield under field conditions. Plant Biotechnology Journal, 10, 792-805.

Reguera, M., Peleg, Z., Abdel-Tawab, Y.M., Tumimbang, E.B., Delatorre, C.A. \& Blumwald, E. (2013) Stress-induced cytokinin synthesis increases drought tolerance through the coordinated regulation of carbon and nitrogen assimilation in rice. Plant Physiology, 163, 1609-1622.

Riera, M., Valon, C., Fenzi, F., Giraudat, J. \& Leung, J. (2005) The genetics of adaptive responses to drought stress: abscisic acid-dependent and abscisic acid-independent signalling components. Physiologia Plantarum, 123, 111-111, 119.

Rohila, J.S., Jain, R.K. \& Wu, R. (2002) Genetic improvement of basmati rice for salt and drought tolerance by regulated expression of a barley HVA1 cDNA. Plant Science, 163, 525-532.

Sahebi, M., Hanafi, M.M., Rafii, M.Y., Mahmud, T.M.M., Azizi, P., Osman, M., et al. (2018) Improvement of drought tolerance in rice (Oryza sativa L.): genetics, genomic tools, and the WRKY gene family. BioMed Research International, 3158474, 1-20.

Saibo, N.J., Lourenço, T. \& Oliveira, M.M. (2009) Transcription factors and regulation of photosynthetic and related metabolism under environmental stresses. Annals of Botany, 103, 609-623.

Sakuma, Y., Maruyama, K., Osakabe, Y., Qin, F., Seki, M., Shinozaki, K., et al. (2006) Functional analysis of an Arabidopsis transcription factor, DREB2A, involved in drought-responsive gene expression. Plant Cell, $18,1292-1309$

Sakurai, J., Ishikawa, F., Yamaguchi, T., Uemura, M. \& Maeshima, M. (2005) Identification of 33 rice aquaporin genes and analysis of their expression and function. Plant \& Cell Physiology, 46, 1568-1577.

Sandhu, N., Dixit, S., Swamy, B.P.M., Raman, A., Kumar, S., Singh, S.P., et al. (2019) Marker assisted breeding to develop multiple stress tolerant varieties for flood and drought prone areas. Rice, 12, 8.

Sandhu, N. \& Kumar, K. (2017) Bridging the rice yield gaps under drought: QTLs, genes, and their use in breeding programs. Agronomy, 7, 27.

Sandhu, N., Raman, K.A., Torres, R.O., Audebert, A., Dardou, A., Kumar, A., et al. (2016) Rice root architectural plasticity traits and genetic regions for adaptability to variable cultivation and stress conditions. Plant Physiology, 171, 2562-2576.

Schroeder, J.I., Allen, G.J., Hugouvieux, V., Kwak, J.M. \& Waner, D. (2001) Guard cell signal transduction. Annual Review of Plant Physiology and Plant Molecular Biology, 52, 627-658.
Selvaraj, M.G., Ishizaki, T., Valencia, M., Ogawa, S., Dedicova, B., Ogata, T., et al. (2017) Overexpression of an Arabidopsis thaliana galactinol synthase gene improves drought tolerance in transgenic rice and increased grain yield in the field. Plant Biotechnology Journal, 15, 1465-1477.

Seo, J.S., Joo, J., Kim, M.-J., Kim, Y.-K., Nahm, B.H., Song, S.I., et al. (2011) OsbHLH148, a basic helix-loop-helix protein, interacts with OsJAZ proteins in a jasmonate signaling pathway leading to drought tolerance in rice. The Plant Journal, 65, 907-921.

Serraj, R. \& Sinclair, T.R. (2002) Osmolyte accumulation: can it really help increase crop yield under drought conditions? Plant, Cell \& Environment, 25, 333-341.

Shan, Q., Wang, Y., Li, J., Zhang, Y., Chen, K., Liang, Z., et al. (2013) Targeted genome modification of crop plants using a CRISPR-Cas system. Nature Biotechnology, 31, 686-688.

Shao, H., Wang, H. \& Tang, X. (2015) NAC transcription factors in plant multiple abiotic stress responses: progress and prospects. Frontiers in Plant Science, 6, 902.

Sharma, L., Dalal, M., Verma, R.K., Kumar, S.V.V., Yadav, S.K., Pushkar, S., et al. (2018) Auxin protects spikelet fertility and grain yield under drought and heat stresses in rice. Environmental and Experimental Botany, 150, 9-24.

Shi, F., Yang, X., Zeng, H., Guo, L., Qiu, D. (2018) Label-free quantitative proteomic analysis revealed a positive effect of ectopic overexpression of PeaT1 from Alternaria tenuissima on rice (Oryza sativa) response to drought. 3 biotech 8: 480

Shi, H., Chen, L., Ye, T., Liu, X., Ding, K.K. \& Chan, Z. (2014) Modulation of auxin content in Arabidopsis confers improved drought stress resistance. Plant Physiology and Biochemistry, 82C, 209-217.

Shim, J.S., Oh, N., Chung, P.J., Kim, Y.S., Choi, Y.D. \& Kim, J.K. (2018) Overexpression of OsNAC14 improves drought tolerance in rice. Frontiers in Plant Science, 9, 310.

Shu, L., Lou, Q., Ma, C., Ding, W., Zhou, J., Wu, J., et al. (2011) Genetic, proteomic and metabolic analysis of the regulation of energy storage in rice seedlings in response to drought. Proteomics, 11, 4122-4138.

Silva, E.C., Nogueira, R.J.M.C., Silva, M.A. \& Albuquerque, M. (2011) Drought stress and plant nutrition. Plant Stress, 5, 32-41.

Sinclair, T.R. (2011) Challenges in breeding for yield increase for drought. Trends in Plant Science, 16, 289-293.

Singh, R.K., Deshmukh, R., Muthamilarasan, M., Rani, R. \& Prasad, M. (2020) Versatile roles of aquaporin in physiological processes and stress tolerance in plants. Plant Physiology and Biochemistry, 149, 178-189.

Sohag, A.A.M., Tahjib-Ul-Arif, M., Brestic, M., Afrin, S., Sakil, M.A., Hossain, M.T., et al. (2020) Exogenous salicylic acid and hydrogen peroxide attenuate drought stress in rice. Plant, Soil and Environment, 66, 7-13.

Song, S.Y., Chen, Y., Chen, J., Dai, X.Y. \& Zhang, W.H. (2011) Physiological mechanisms underlying OsNAC5-dependent tolerance of rice plants to abiotic stress. Planta, 234, 331-345.

Srivasta, A., Mehta, S., Lindlof, A. \& Bhargava, S. (2010) Over-represented promoter motifs in abiotic stress-induced DREB genes of rice and sorghum and their probable role in regulation of gene expression. Plant Signaling \& Behavior, 5, 775-784.

Steele, K.A., Price, A.H., Shashidhar, H.E. \& Witcombe, J.R. (2006) Markerassisted selection to introgress rice QTLs controlling root traits into an Indian upland rice variety. Theoretical and Applied Genetics, 112, 208-221.

Steele, K.A., Virk, D.S., Kumar, R., Prasad, S.C. \& Witcombe, J.R. (2007) Field evaluation of upland rice lines selected for QTLs controlling root traits. Field Crops Research, 101, 180-186.

Sun, L.R., Yue, C.M. \& Hao, F.S. (2019) Update on roles of nitric oxide in regulating stomatal closure. Plant Signaling \& Behavior, 14, e1649569.

Suzuki, N., Taketa, S. \& Ichii, M. (2003) Morphological and physiological characteristics of a root-hairless mutant in rice (Oryza sativa L.). Plant and Soil, 255, 9-17. 
Svitashev, S., Young, J.K., Schwartz, C., Gao, H., Falco, S.C. \& Cigan, A.M. (2015) Targeted mutagenesis, precise gene editing, and site-specific gene insertion in maize using Cas9 and guide RNA. Plant Physiology, 169, 931-945.

Swain, P., Anumalla, M., Prusty, S., Marndi, B.C. \& Rao, G.J.N. (2014) Characterization of some Indian native land race rice accessions for drought tolerance at seedling stage. Australian Journal of Crop Science, 8, 324-331.

Swamy, B.P., Ahmed, H.U., Henry, A., Mauleon, R., Dixit, S., Vikram, P., et al. (2013) Genetic, physiological, and gene expression analyses reveal that multiple QTL enhance yield of rice mega-variety IR64 under drought. PLoS One, 8, e62795.

Swamy, B.P.M. \& Kumar, A. (2012) Sustainable rice yield in watershort drought-prone environments. In: Irrigation Systems and Practices in Challenging Environments, Teang Shui Lee, IntechOpen. https://doi. org/10.5772/30696.

Takahashi, Y., Zhang, J., Hsu, P.K., Ceciliato, P.H.O., Zhang, L., Dubeaux, G., et al. (2020) MAP3Kinase-dependent SnRK2-kinase activation is required for abscisic acid signal transduction and rapid osmotic stress response. Nature Communications, 11, 12.

Tang, Y., Bao, X., Zhi, Y., Wu, Q., Yin, X., Zeng, L., et al. (2019) Overexpression of a MYB family gene, OsMYB6, increases drought and salinity stress tolerance in transgenic rice. Frontiers in Plant Science, 10, 168.

Tian, X., Wang, Z., Li, X., Lv, T., Liu, H., Wang, L., et al. (2015) Characterization and functional analysis of Pyrabactin resistance-like abscisic acid receptor family in rice. Rice, 8, 28.

Tran, T.T., Kano-Nakata, M., Suralta, R.R., Menge, D., Mitsuya, S., Inukai, Y., et al. (2015) Root plasticity and its functional roles were triggered by water deficit but not by the resulting changes in the forms of soil $\mathrm{N}$ in rice. Plant and Soil, 386, 65-76.

Uga, Y., Okuno, K. \& Yano, M. (2011) Dro1, a major QTL involved in deep rooting of rice under upland field conditions. Journal of Experimental Botany, 62, 2485-2494.

Uga, Y., Sugimoto, K., Ogawa, S., Rane, J., Ishitani, M., Hara, N., et al. (2013) Control of root system architecture by DEEPER ROOTING 1 increases rice yield under drought conditions. Nature Genetics, 45, 1097-1102.

UI Haq, S., Khan, A., Ali, M., Khattak, A.M., Gai, W.X., Zhang, Z.-X., et al. (2019) Heat shock proteins: dynamic biomolecules to counter plant biotic and abiotic stresses. International Journal of Molecular Sciences, 20, 5321.

Umezawa, T., Nakashima, K., Miyakawa, T., Kuromori, T., Tanokura, M., Shinozaki, K., et al. (2010) Molecular basis of the core regulatory network in ABA responses: sensing, signaling and transport. Plant \& Cell Physiology, 51, 1821-1839.

Venuprasad, R., Dalid, C.O., Del Valle, M., Zhao, D., Espiritu, M., Sta Cruz, M.T., et al. (2009) Identification and characterization of largeeffect quantitative trait loci for grain yield under lowland drought stress in rice using bulk-segregant analysis. Theoretical and Applied Genetics, 120, 177-190.

Venuprasad, R., Lafitte, H.R. \& Atlin, G.N. (2007) Response to direct selection for grain yield under drought stress in rice. Crop Science, 47, 285-293.

Verma, H., Borah, J.L. \& Sarma, R.N. (2019) Variability assessment for root and drought tolerance traits and genetic diversity analysis of rice germplasm using SSR markers. Scientific Reports, 9, 16513.

Vikram, P., Swamy, B.P., Dixit, S., Ahmed, H.U., Teresa Sta Cruz, M., Singh, A.K., et al. (2011) qDTY1.1, a major QTL for rice grain yield under reproductive-stage drought stress with a consistent effect in multiple elite genetic backgrounds. BMC Genetics, 12, 89.

Vinod, K.K., Krishnan, S.G., Thribhuvan, R. \& Singh, A.K. (2019) Genetics of drought tolerance, mapping QTLs, candidate genes and their utilization in rice improvement. In: Rajpal, V., Sehgal, D., Kumar, A. \& Raina, S. (Eds.) Genomics assisted breeding of crops for abiotic stress tolerance,
Vol. II. Sustainable development and biodiversity, Vol. 21. Cham: Springer.

Vishwakarma, K., Upadhyay, N., Kumar, N., Yadav, G., Singh, J., Mishra, R. K., et al. (2017) Abscisic acid signaling and abiotic stress tolerance in plants: a review on current knowledge and future prospects. Frontiers in Plant Science, 8, 161.

Wan, L., Zhang, J., Zhang, H., Zhang, Z., Quan, R., Zhou, S., et al. (2011) Transcriptional activation of OsDERF1 in OsERF3 and OsAP2-39 negatively modulates ethylene synthesis and drought tolerance in rice. PLoS One, 6, e25216.

Wang, B., Zhong, Z., Wang, X., Han, X., Yu, D., Wang, C., et al. (2020b) Knockout of the OsNACOO6 transcription factor causes drought and heat sensitivity in rice. International Journal of Molecular Sciences, 21, 2288.

Wang, C., Chen, S., Dong, Y., Ren, R., Chen, D. \& Chen, X. (2020a) Chloroplastic Os3BGlu6 contributes significantly to cellular ABA pools and impacts drought tolerance and photosynthesis in rice. The New Phytologist, 226, 1042-1054.

Wang, M., Zheng, Q.S., Shen, Q.R. \& Guo, S.W. (2013) The critical role of potassium in plant stress response. International Journal of Molecular Sciences, 14, 7370-7390.

Wang, Q., Guan, Y., Wu, Y., Chen, H., Chen, F. \& Chu, C. (2008) Overexpression of a rice OsDREB1F gene increases salt, drought, and low temperature tolerance in both Arabidopsis and rice. Plant Molecular Biology, 67, 589-602.

Wang, X., Cai, X., Xu, C., Wang, Q. \& Dai, S. (2016) Drought-responsive mechanisms in plant leaves revealed by proteomics. International Journal of Molecular Sciences, 17, 1706.

Wang, X., Li, B.B., Ma, T.T., Sun, L.Y., Tai, L., Hu, C.H., et al. (2020c) The NAD kinase OsNADK1 affects the intracellular redox balance and enhances the tolerance of rice to drought. BMC Plant Biology, 20, 1-19.

Wu, J., Kim, S.G., Kang, K.Y., Kim, J.G., Park, S.R., Gupta, R., et al. (2016b) Overexpression of a pathogenesis-related protein 10 enhances biotic and abiotic stress tolerance in Rice. Plant Pathology Journal, 32, 552-562.

Wu, Y., Mirzaei, M., Pascovici, D., Chick, J.M., Atwell, B.J. \& Haynes, P.A. (2016a) Quantitative proteomic analysis of two different rice varieties reveals that drought tolerance is correlated with reduced abundance of photosynthetic machinery and increased abundance of ClpD1 protease. Journal of Proteomics, 143, 73-82.

Xiao, B., Huang, Y., Tang, N. \& Xiong, L. (2007) Over-expression of a LEA gene in rice improves drought resistance under the field conditions. Theoretical and Applied Genetics, 115, 35-46.

Xie, W., Wang, G., Yuan, M., Yao, W., Lyu, K., Zhao, H., et al. (2015) Breeding signatures of rice improvement revealed by a genomic variation map from a large germplasm collection. Proceedings of the National Academy of Sciences of the United States of America, 112, E5411-E5419.

Xiong, H., Yu, J., Miao, J., Li, J., Zhang, H., Wang, X., et al. (2018) Natural variation in OsLG3 increases drought tolerance in rice by inducing ROS scavenging. Plant Physiology, 178, 451-467.

Xiong, Q., Cao, C., Shen, T., Zhong, L., He, H.H. \& Chen, X. (2019a) Comprehensive metabolomic and proteomic analysis in biochemical metabolic pathways of rice spikes under drought and submergence stress. Biochimica et Biophysica Acta (BBA) - Proteins and Proteomics, 1867, 237-247.

Xiong, Q., Shen, T., Zhong, L., Zhu, C.L., Peng, X.-S., He, X.-P., et al. (2019b) Comprehensive metabolomic, proteomic and physiological analyses of grain yield reduction in rice under abrupt drought-flood alternation stress. Physiologia Plantarum, 167, 564-584.

Xu, W., Cui, K., Xu, A., Nie, L., Huang, J. \& Peng, S. (2015) Drought stress condition increases root to shoot ratio via alteration of carbohydrate partitioning and enzymatic activity in rice seedlings. Acta Physiologiae Plantarum, 37, 1760. 
Xu, W., Jia, L., Shi, W., Liang, J., Zhou, F., Li, Q., et al. (2013) Abscisic acid accumulation modulates auxin transport in the root tip to enhance proton secretion for maintaining root growth under moderate water stress. The New Phytologist, 197, 139-150.

Xue, T., Wang, D., Zhang, S., Ehlting, J., Ni, F., Jakab, S., et al. (2008) Genome-wide and expression analysis of protein phosphatase $2 \mathrm{C}$ in rice and Arabidopsis. BMC Genomics, 9, 550.

Yaish, M.W., El-Kereamy, A., Zhu, T., Beatty, P.H., Good, A.G., Bi, Y.M., et al. (2010) The APETALA-2-like transcription factor OsAP2-39 controls key interactions between abscisic acid and gibberellin in rice. PLoS Genetics, 6, e1001098.

Yamaguchi-Shinozaki, K. \& Shinozaki, K. (2005) Organization of cis-acting regulatory elements in osmotic- and cold-stress-responsive promoters. Trends in Plant Science, 10, 88-94.

Yambao, E.B. \& O'Toole, J.C. (1984) Effects of nitrogen nutrition and root medium water potential on growth, nitrogen uptake and osmotic adjustment of rice. Physiologia Plantarum, 60, 507-515.

Yang, A., Dai, X. \& Zhang, W.H. (2012b) A R2R3-type MYB gene, OsMYB2, is involved in salt, cold, and dehydration tolerance in rice. Journal of Experimental Botany, 63, 2541-2556.

Yang, J., Zhang, J., Liu, K., Wang, Z. \& Liu, L. (2007) Abscisic acid and ethylene interact in rice spikelets in response to water stress during meiosis. Journal of Plant Growth Regulation, 26, 318-328.

Yang, S., Xu, K., Chen, S., Li, T., Xia, H., Chen, L., et al. (2019) A stressresponsive bZIP transcription factor OsbZIP62 improves drought and oxidative tolerance in rice. BMC Plant Biology, 19, 260.

Yang, T., Zhang, S., Hu, Y., Wu, F., Hu, Q., Chen, G., et al. (2014) The role of a potassium transporter OsHAK5 in potassium acquisition and transport from roots to shoots in rice at low potassium supply levels. Plant Physiology, 166, 945-959.

Yang, X., Li, Y., Ren, B., Ding, L., Gao, C., Shen, Q., et al. (2012a) Droughtinduced root aerenchyma formation restricts water uptake in rice seedlings supplied with nitrate. Plant \& Cell Physiology, 53, 495-504.

Yao, L., Cheng, X., Gu, Z., Huang, W., Li, S., Wang, L., et al. (2018) The AWPM-19 family protein OsPM1 mediates abscisic acid influx and drought response in rice. Plant Cell, 30, 1258-1276.

Ye, N., Jia, L. \& Zhang, J. (2012) ABA signal in rice under stress conditions. Rice, 5, 1.

You, J., Hu, H. \& Xiong, L. (2012) An ornithine $\delta$-aminotransferase gene OsOAT confers drought and oxidative stress tolerance in rice. Plant Science, 197, 59-69.

You, J., Zong, W., Hu, H., Li, X., Xiao, J. \& Xiong, L. (2014) A stressresponsive NAC1-regulated protein phosphatase gene rice protein phosphatase18 modulates drought and oxidative stress tolerance through abscisic acid-independent reactive oxygen species scavenging in rice. Plant Physiology, 166, 2100-2114.

You, J., Zong, W., Li, X., Ning, J., Hu, H., Li, X., et al. (2013) The SNAC1-targeted gene OsSRO1c modulates stomata closure and oxidative stress tolerance by regulating hydrogen peroxide in rice. Journal of Experimental Botany, 64, 569-583.

Yu, J., Hu, S., Wang, J., Wong, G., Li, S., Liu, B., et al. (2002) A draft sequence of the rice genome (Oryza sativa L. ssp. indica). Science, 296, 79-92.
Yu, Y., Yang, D., Zhou, S., Gu, J., Wang, F., Dong, J., et al. (2017) The ethylene response factor OsERF109 negatively affects ethylene biosynthesis and drought tolerance in rice. Protoplasma, 254, 401-408.

Yuan, X., Huang, P., Wang, R., Li, H., Lv, X., Duan, M., et al. (2018) A zinc finger transcriptional repressor confers pleiotropic effects on rice growth and drought tolerance by down-regulating stress-responsive genes. Plant \& Cell Physiology, 59, 2129-2142.

Zhang, C., Li, C., Liu, J., Lv, Y., Yu, C., Li, H., et al. (2017) The OsABF1 transcription factor improves drought tolerance by activating the transcription of COR413-TM1 in rice. Journal of Experimental Botany, 68, 4695-4707.

Zhang, F., Luo, X., Zhou, Y. \& Xie, J. (2015) Genome-wide identification of conserved microRNA and their response to drought stress in Dongxiang wild rice (Oryza rufipogon Griff.). Biotechnology Letters, 38, 711-721.

Zhang, H., Zhang, J., Quan, R., Pan, X., Wan, L. \& Huang, R. (2013) EAR motif mutation of rice OsERF3 alters the regulation of ethylene biosynthesis and drought tolerance. Planta, 237, 1443-1451.

Zhang, Q., Li, J., Zhang, W., Yan, S., Wang, R., Zhao, J., et al. (2012) The putative auxin efflux carrier OsPIN3t is involved in the drought stress response and drought tolerance. The Plant Journal, 72, 805-816.

Zhang, S.-W., Li, C.H., Cao, J., Zhang, Y.-C., Zhang, S.-Q., Xia, Y.-F., et al. (2009) Altered architecture and enhanced drought tolerance in rice via the down-regulation of indole-3-acetic acid by TLD1/OsGH3. 13 activation. Plant Physiology, 151, 1889-1901.

Zhang, Y., Li, J., Chen, S., Ma, X., Wei, H., Chen, C., et al. (2020b) An APETALA2/ethylene responsive factor, OsEBP89 knockout enhances adaptation to direct-seeding on wet land and tolerance to drought stress in rice. Molecular Genetics and Genomics, 295, 941-956.

Zhang, Y., Wang, X., Luo, Y., Zhang, L., Yao, Y., Han, L., et al. (2020a) OsABA8ox2, an ABA catabolic gene, suppresses root elongation of rice seedlings and contributes to drought response. Crop Journal, 8, 480-491.

Zhang, Z., Li, F., Li, D., Zhang, H. \& Huang, R. (2010) Expression of ethylene response factor JERF1 in rice improves tolerance to drought. Planta, 232, 765-774.

Zhao, H., Ma, T., Wang, X., Deng, Y., Ma, H., Zhang, R., et al. (2015) OsAUX 1 controls lateral root initiation in rice (Oryza sativa L.). Plant, Cell \& Environment, 38, 2208-2222.

Zhu, J., Song, N., Sun, S., Yang, W., Zhao, H., Song, W., et al. (2016) Efficiency and inheritance of targeted mutagenesis in maize using CRISPR-Cas9. Journal of Genetics and Genomics, 43, 25-36.

\section{SUPPORTING INFORMATION}

Additional supporting information may be found online in the Supporting Information section at the end of this article.

How to cite this article: Khan MIR, Palakolanu SR, Chopra P, et al. Improving drought tolerance in rice: Ensuring food security through multi-dimensional approaches. Physiologia

Plantarum. 2020;1-24. https://doi.org/10.1111/ppl.13223 